\title{
Global weak solutions in a PDE-ODE system modeling multiscale cancer cell invasion
}

\author{
Christian Stinner $^{1 *} \quad$ Christina Surulescu ${ }^{1 \#}$ \\ Michael Winkler ${ }^{2 \ddagger}$ \\ 1 Technische Universität Kaiserslautern, Felix-Klein-Zentrum für Mathematik, \\ Paul-Ehrlich-Str. 31, 67663 Kaiserslautern, Germany \\ ${ }^{2}$ Institut für Mathematik, Universität Paderborn, 33098 Paderborn, Germany
}

\begin{abstract}
We prove the global existence, along with some basic boundedness properties, of weak solutions to a PDE-ODE system modeling the multiscale invasion of tumor cells through the surrounding tissue matrix. The model has been proposed in [22] and accounts on the macroscopic level for the evolution of cell and tissue densities, along with the concentration of a chemoattractant, while on the subcellular level it involves the binding of integrins to soluble and insoluble components of the peritumoral region. The connection between the two scales is realized with the aid of a contractivity function characterizing the ability of the tumor cells to adapt their motility behavior to their subcellular dynamics.

The resulting system, consisting of three partial and three ordinary differential equations including a temporal delay, in particular involves chemotactic and haptotactic cross-diffusion. In order to overcome technical obstacles stemming from the corresponding highest-order interaction terms, we base our analysis on a certain functional, inter alia involving the cell and tissue densities in the diffusion and haptotaxis terms respectively, which is shown to enjoy a quasi-dissipative property. This will be used as a starting point for the derivation of a series of integral estimates finally allowing for the construction of a generalized solution as the limit of solutions to suitably regularized problems.
\end{abstract}

Key words: chemotaxis; haptotaxis; asymptotic behavior; multiscale model; delay.

AMS Classification: 35Q92, 92C17, 35K57, 35B40.

\footnotetext{
*stinner@mathematik.uni-kl.de

\# surulescu@mathematik.uni-kl.de

${ }^{\ddagger}$ michael.winkler@math.uni-paderborn.de
} 


\section{Introduction}

The model. The migration of cancer cells from the original tumor toward blood vessels and the subsequent transport to distal sites eventually leading to metastasis establishment is influenced by a plethora of biochemical processes [8]. These take place at several scales, ranging from the level of subcellular events and up to the macroscopic behavior of tissue and cell populations. Thereby, the time scales spread from seconds (or less) for the processes inside cells or on their surface, up to months for the doubling times of tumors or for the time it takes to develop clinically detectable secondary tumors.

Chemotaxis and haptotaxis are two of the main mechanisms conditioning cancer cell motility. The former terms the directed cell motion in response to concentration gradients of some chemoattractant/chemorepellent. The latter refers to changes in the orientation and speed of the cell according to interactions (mainly adhesion) with the fibers of the extracellular matrix (ECM): The cells will migrate from a region of low concentration of relevant adhesive molecules towards a region with a higher concentration. Mathematical models set up on this macrolevel and involving at least one of these two mechanisms have been proposed and analyzed in literature, see e.g., [2, 34] and the references therein.

The macroscopic features mentioned above are, however, influenced by the internal state of cells, hence by microscopic processes, e.g., receptor binding to chemoattractant or adhesion molecules initiating intracellular signaling pathways, which in turn lead to restructuring of the cytoskeleton, polarization, production of matrix degrading enzymes (MDEs), proliferation, etc. [7, 13, 14]. Including this microlevel dynamics into the mathematical model leads to a multiscale setting coupling a system of partial differential equations (PDEs) for the quantities on the macroscale with ordinary differential equations (ODEs) modeling the subcellular events. These continuum micro-macro models explicitly accounting for subcellular events are rather new, especially in the context of cancer cell migration. Other (mainly individual based) multiscale models connecting the macrolevel with the subcellular and/or cellular scale have been proposed e.g., by Macklin et al. [19] for vascular tumor growth also involving mechanic effects or by Ramis-Conde et al. [27, 28], where an individual force-based approach is considered, focusing on the influence of intra- and intercellular dynamics on the cell movement. Hybrid models using cellular automata or agent-based approaches (see e.g., [9, 39, 44]) provide a natural framework for coupling individual events with macrolevel features, thereby allowing for a high level of detail. However, when the number of cells greatly increases to biologically realistic values this approach can become very expensive or even intractable from a computational point of view. In contrast, continuum models describe the evolution of population-averaged phenomena and are more efficient from a numerical point of view. In this framework Webb et al. [40] studied a population based micro-macro model coupling the tumor cell and ECM densities with concentrations of MMP (matrix metalloproteinases) and protons, the latter both in their intracellular and extracellular forms. Mallet \& Pettet [20] proposed a model for the integrin-mediated haptotactic migration, which assigns particular attention to the role played by cell surface receptors in the adhesion to ECM.

All previously mentioned settings focused on the modeling and illustration of the involved dynamics by way of numerical simulations, paying less attention to the mathematical well posedness. The latter is a nontrivial issue, due to the fact that in the multiscale models different types of equations are coupled in a highly nonlinear way and the regularity assumptions which can be made are rather modest, according to biologically motivated requirements. Also, the models are sensitive to changes 
in the initial data and in the parameter ranges, which in turn are difficult to assess in the absence of adequate quantitative information. Hence, the solutions to the corresponding systems can exhibit oscillatory behavior and even blow-up. Such problems are often encountered in chemotaxis systems (see e.g., $[3,5])$. Global existence of solutions to systems modeling tumor invasion has been investigated among others by Liţcanu \& Morales-Rodrigo [17] and Tao [30] (accounting for haptotaxis only) and by Tao et al. [29, 31, 32, 34] (chemotaxis and haptotaxis jointly influencing the motion). The analyzed models were set on the macroscale and described the evolution of tumor cells in interaction with the surrounding tissue and MDEs, hence coupling reaction-diffusion and ordinary differential equations in two or three space dimensions. In the context of multiscale cancer cell migration the well posedness issue was addressed e.g., in [15, 18] (micro-meso-macro models) and in [22, 23] (micro-macro models), respectively. The present work is concerned with this problem, too. Here the subcellular (microlevel) dynamics is represented by the binding of integrins to the respective ligands and this is seen as the onset of all subsequent intracellular signaling and its effects on the cell responses. The coupling with the macrolevel is realized by way of a contractivity function capturing the effects of subcellular dynamics on the cell motility and carrying them over into the diffusion and haptotaxis coefficients of the corresponding terms in the equation describing the evolution of tumor cells. This function satisfies itself a differential equation involving the concentrations of bound integrins as inputs. As the conversion of biochemical signals initiated by integrin binding into motile behavior needs a long sequence of events to happen, we allow for a time lag in the contractivity equation, which hence takes the form of a delay differential equation (DDE). We recall here the micro-macro setting in [22] and refer to that work for the modeling details. Upon non-dimensionaliziation, the model proposed there becomes

$$
\left\{\begin{array}{l}
\partial_{t} c=\nabla \cdot\left(\frac{\kappa}{1+c v} \nabla c\right)-\nabla \cdot\left(\frac{\kappa v}{1+v} c \nabla v\right)-\nabla \cdot\left(\frac{c}{1+c l} \nabla l\right)+\mu_{c} c\left(1-c-\eta_{1} v\right), \quad x \in \Omega, t>0, \\
\partial_{t} v=\mu_{v} v(1-v)-\lambda c v, \quad x \in \Omega, t>0, \\
\partial_{t} l=\Delta l-l+c v, \quad x \in \Omega, t>0, \\
\partial_{t} y_{1}=k_{1}\left(1-y_{1}-y_{2}\right) v-k_{-1} y_{1}, \quad x \in \Omega, t>0, \\
\partial_{t} y_{2}=k_{2}\left(1-y_{1}-y_{2}\right) l-k_{-2} y_{2}, \quad x \in \Omega, t>0, \\
\partial_{t} \kappa=-\kappa+\frac{M y_{1}(\cdot, t-\tau)}{1+y_{2}(\cdot, t-\tau)}, \quad x \in \Omega, t>0,
\end{array}\right.
$$

the dependent variables respectively representing the following:

- $c(x, t)$ : cancer cell density;

- $v(x, t)$ : density of tissue fibers in the ECM;

- $l(x, t)$ : concentration of chemoattractant (i.e., proteolytic rests resulting from degradation of ECM fibers by matrix degrading enzymes produced by the tumor cells);

- $y_{1}(x, t)$ : concentration of integrins bound to ECM fibers;

- $y_{2}(x, t)$ : concentration of integrins bound to proteolytic residuals;

- $\kappa(x, t)$ : contractivity function;

- $\mu_{c}$ : proliferation rate of tumor cells; 
- $\mu_{v}$ : production/re-establishment rate of ECM fibers;

- $\delta$ : degradation rate of ECM fibers due to the action of MDEs;

- $\eta_{1}, \eta_{2}$ : interaction rates allowing to take crowding into account;

- $k_{-1}, k_{1}, k_{-2}, k_{2}$ : reaction rates in the binding of integrins to insoluble (ECM fibers) and soluble (proteolytic rests) ligands in the peritumoral environment.

Moreover, we abbreviate $\lambda:=\delta+\mu_{v} \eta_{2}$.

Mathematical challenges. The goal of the present work is to establish a result on global solvability of (1.1) in an adequate framework. From a mathematical point of view, this amounts to appropriately overcome several difficulties inherent to (1.1) and, in particular, the strong couplings therein. Indeed, as a subsystem the model (1.1) contains a Keller-Segel-type chemotaxis system, the original form of which, that is,

$$
\left\{\begin{array}{l}
\partial_{t} c=\Delta c-\nabla \cdot(c \nabla l), \quad x \in \Omega, t>0, \\
\partial_{t} l=\Delta l-l+c, \quad x \in \Omega, t>0
\end{array}\right.
$$

is known to exhibit phenomena of blow-up in finite time when either $n \geq 3$ (see [43]) or $n=2$ and the total mass of cells is supercritical $[10,24]$. As compared to $(1.2)$, both the diffusivity $D(c, v):=\frac{\kappa}{1+c v}$ and the chemotactic sensitivity $\chi(c, l):=\frac{c}{1+c l}$ in (1.1) depend nonlinearly on $c, v$, and $l$, thus leading to an even higher complexity of interaction. In [4] it has been shown that in the neighboring case when $D=D(c)=\frac{1}{1+c}$ and $\chi=\chi(c)=\frac{c}{1+c}$, blow-up in the correspondingly adapted variant of (1.2) does as well occur at least when $n=3$. Anyhow, the logistic-type cell kinetic term in the first equation in (1.1), is known to have a certain blow-up preventing effect on chemotaxis systems: When accordingly introduced in (1.2), for instance, proliferation terms of the form $\mu_{c} c(1-c)$ are known to enforce boundedness of solutions when $n \leq 2$ (see [25]), and also when $n \geq 3$ and $\mu_{c}$ is suitably large [42]; however, this apparently does not exclude the possibility of explosions when $n=3$ and $\mu_{c}$ is small.

A second subsystem is obtained upon focusing on the cross-diffusive interaction with $v$, resulting in a variant of the prototypical haptotaxis system

$$
\left\{\begin{array}{l}
\partial_{t} c=\Delta c-\nabla \cdot(c \nabla v), \quad x \in \Omega, t>0, \\
\partial_{t} v=f(c, v), \quad x \in \Omega, t>0,
\end{array}\right.
$$

with given $f$. Here the lack of diffusibility of $v$ is reflected in the absence of any regularizing effect on $v$ during evolution. Correspondingly, the mathematical literature on (1.3) is comparatively thin, the main contributions on haptotaxis systems concentrating on particular choices of $f[6,38]$ or on modified variants involving additional dampening effects such as logistic growth inhibition [21, 17]. More recently, certain combined chemotaxis-haptotaxis models with linear cell diffusion and standard cross-diffusive terms as in (1.2) and (1.3), have been investigated, and some results on global existence and also on asymptotic solution behavior could be gained for various special choices of $f$ in the respective ODE $\partial_{t} v=f(c, v, l)[29,33,35,36,12]$.

Main results. In the sequel, we shall consider the PDE-ODE system (1.1) in a bounded domain 
$\Omega \subset \mathbb{R}^{n}, n \leq 3$, with smooth boundary, under no-flux boundary conditions ( $\nu$ denotes the outer normal to $\partial \Omega$ ):

$$
\frac{\kappa}{1+c v} \partial_{\nu} c-\frac{\kappa v}{1+v} c \partial_{\nu} v=\partial_{\nu} l=0, \quad x \in \partial \Omega, t>0
$$

and the initial conditions

$$
\begin{aligned}
& c(x, 0)=c_{0}(x), \quad v(x, 0)=v_{0}(x), \quad l(x, 0)=l_{0}(x), \quad \kappa(x, 0)=\kappa_{0}(x), \quad x \in \Omega, \\
& y_{1}(x, t)=y_{10}(x, t), \quad y_{2}(x, t)=y_{20}(x, t), \quad x \in \Omega, t \in[-\tau, 0],
\end{aligned}
$$

where we assume that

$$
\begin{aligned}
& c_{0} \in C^{0}(\bar{\Omega}), \quad v_{0} \in W^{1,2}(\Omega) \cap C^{0}(\bar{\Omega}), \quad l_{0} \in W^{1,2}(\Omega) \cap C^{0}(\bar{\Omega}), \quad \kappa_{0} \in W^{1,4}(\Omega), \\
& y_{10} \in C^{0}\left([-\tau, 0] ; W^{1,4}(\Omega)\right) \text { and } y_{20} \in C^{0}\left([-\tau, 0] ; W^{1,4}(\Omega)\right)
\end{aligned}
$$

satisfy

$$
\begin{array}{lcc}
c_{0} \geq 0, \quad v_{0} \geq 0, \quad l_{0}>0, \quad \kappa_{0}>0 \quad \text { in } \bar{\Omega} & \text { as well as } \\
y_{10} \geq 0, \quad y_{20} \geq 0 \quad \text { and } \quad y_{10}+y_{20} \leq 1 \quad \text { in } \Omega \times(-\tau, 0) .
\end{array}
$$

The parameters $\mu_{c}, \eta_{1}, \mu_{v}, \lambda:=\delta+\mu_{v} \eta_{2}, k_{1}, k_{-1}, k_{2}, k_{-2}, M$ and $\tau$ are assumed to be positive throughout.

In this mathematical setting, the main outcome of this paper then establishes the global existence of solutions in an appropriate generalized sense, along with some basic global qualitative properties. Besides providing accessibility to our mathematical approach described below, such a resort to weak solutions may be adequate also from a biological point of view: As cell migration is a process featured by very complex phenomena and influenced by a strongly heterogeneous and rapidly modifying environment, the cell and fiber densities - though existing globally in time - should be allowed to be less regular and the conditions for their existence to be less restrictive than, for instance, required in frameworks of classical solutions.

In its precise formulation, our main result reads as follows.

Theorem 1.1 Let $n \leq 3$ and $\Omega \subset \mathbb{R}^{n}$ be a bounded domain with smooth boundary, and suppose that $c_{0}, v_{0}, l_{0}, y_{10}, y_{20}$ and $\kappa_{0}$ comply with (1.6) and (1.7). Then (1.1), (1.4), (1.5) possesses at least one global weak solution in the sense specified in Definition 5.1 below. Moreover, this solution has the following global boundedness properties: There exists $C>0$ such that

$$
\left\{\begin{array}{l}
\int_{\Omega} c(\cdot, t) \leq C \quad \text { and } \quad \int_{t}^{t+1} \int_{\Omega} c^{2} \leq C \quad \text { for all } t>0, \\
v \leq C \quad \text { in } \Omega \times(0, \infty), \\
\|l(\cdot, t)\|_{W^{1,2}(\Omega)} \leq C \quad \text { and } \quad \int_{t}^{t+1}\|l(\cdot, s)\|_{W^{2,2}(\Omega)}^{2} d s \leq C \quad \text { for all } t>0, \\
y_{1} \leq C \quad \text { in } \Omega \times(0, \infty), \\
y_{2} \leq C \quad \text { in } \Omega \times(0, \infty) \\
\kappa \leq C \quad \text { in } \Omega \times(0, \infty) .
\end{array}\right.
$$


Main ideas underlying our approach. A cornerstone of our analysis will consist of identifying a certain quasi-dissipative property of the functional

$$
\mathcal{E}:=\int_{\Omega} c \ln c+\frac{1}{2 \lambda} \int_{\Omega} \frac{\kappa|\nabla v|^{2}}{1+v} .
$$

Indeed, we shall see in Lemma 4.1 below that a certain regularized variant thereof satisfies an inequality of the form

$$
\frac{d}{d t} \mathcal{E}+\mathcal{D} \leq F(t) \mathcal{E}+H(t-\tau), \quad t>0
$$

with some nonnegative $\left.F \in L_{l o c}^{1}[0, \infty)\right)$, a function $H \in L_{l o c}^{1}([0, \infty))$ allowing for the estimate

$$
H(t-\tau) \leq C \mathcal{E}(t-\tau) \quad \text { for all } t>\tau
$$

with some $C>0$, and with the dissipation rate given by

$$
\mathcal{D}:=\frac{1}{2} \int_{\Omega} \frac{\kappa}{1+c v} \frac{|\nabla c|^{2}}{c}+\int_{\Omega} \kappa c \frac{|\nabla v|^{2}}{(1+v)^{2}}+\frac{\mu_{c}}{2} \int_{\Omega} c^{2} \ln (2+c)
$$

(cf. (4.31)). In particular, this will imply an integral estimate of the form

$$
\mathcal{E}(t) \leq C(T)+C(T) \int_{0}^{t} \mathcal{E}(\sigma) d \sigma \quad \text { for all } t>0
$$

with suitably large $C>0$ (see (4.36)), whereupon the Grønwall lemma will provide bounds for

$\sup _{t \in(0, T)} \mathcal{E}(t)$ and $\int_{0}^{T} \mathcal{D}(t) d t$ for any fixed $T>0$. These in turn will form the fundament for our derivation of appropriate compactness properties of solutions to the regularized problems (2.1) below (see Section 5.1), and thereby finally allow for the construction of weak solutions as limits of global smooth solutions to these approximate problems in Section 5.2.

Finally, in Section 6 we will comment on some possible model extensions.

\section{A family of approximate problems}

In order to construct a solution of (1.1), (1.4), (1.5), let us first consider the regularized problems

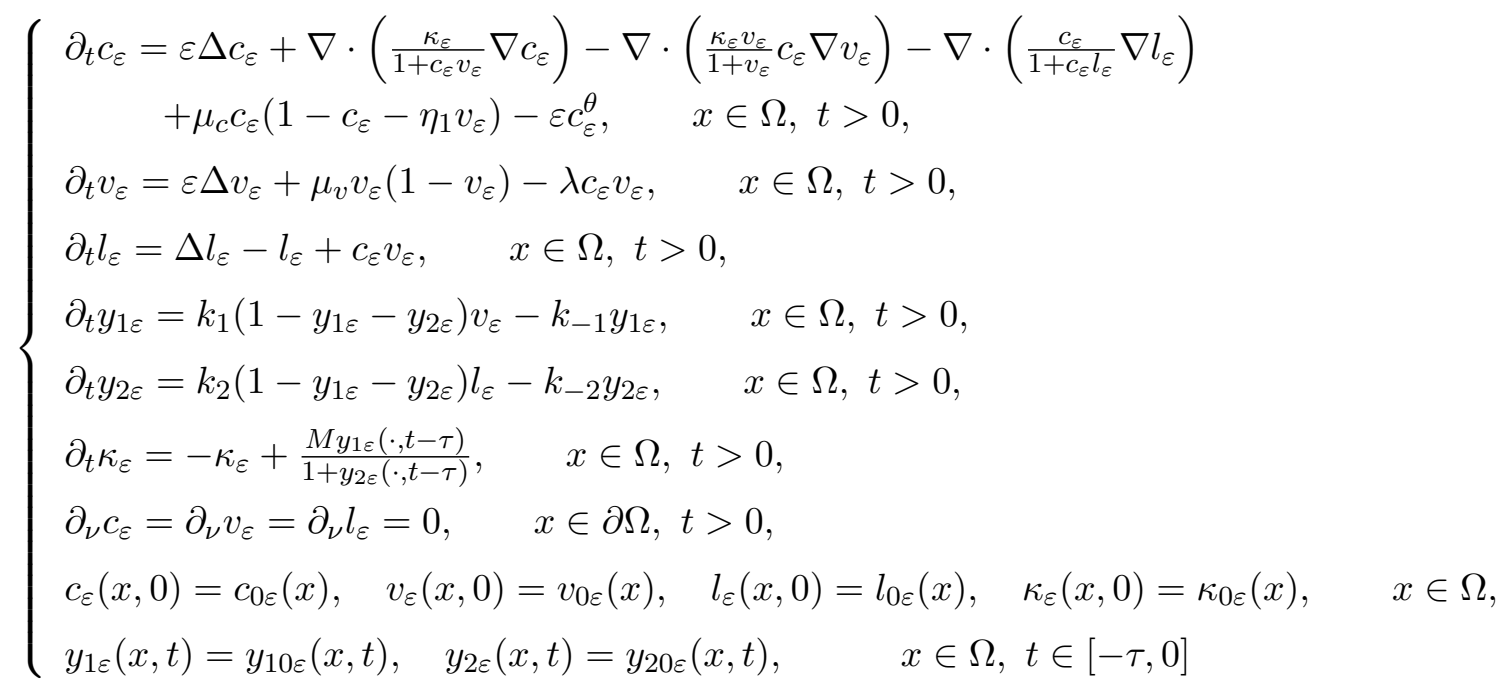


for $\varepsilon \in(0,1)$, where $\theta>\max \{2, n\}$ is a fixed parameter and $\left(c_{0 \varepsilon}\right)_{\varepsilon \in(0,1)},\left(v_{0 \varepsilon}\right)_{\varepsilon \in(0,1)}, \quad\left(l_{0 \varepsilon}\right)_{\varepsilon \in(0,1)}$, $\left(\kappa_{0 \varepsilon}\right)_{\varepsilon \in(0,1)},\left(y_{10 \varepsilon}\right)_{\varepsilon \in(0,1)}$ and $\left(y_{20 \varepsilon}\right)_{\varepsilon \in(0,1)}$ are families of functions

$$
\begin{aligned}
& c_{0 \varepsilon} \in C^{3}(\bar{\Omega}), \quad v_{0 \varepsilon} \in C^{3}(\bar{\Omega}), \quad l_{0 \varepsilon} \in C^{3}(\bar{\Omega}), \quad \kappa_{0 \varepsilon} \in C^{3}(\bar{\Omega}), \\
& y_{10 \varepsilon} \in C^{3}(\bar{\Omega} \times[-\tau, 0]) \quad \text { and } \quad y_{20 \varepsilon} \in C^{3}(\bar{\Omega} \times[-\tau, 0])
\end{aligned}
$$

which are all positive and satisfy

$$
\partial_{\nu} c_{0 \varepsilon}=\partial_{\nu} v_{0 \varepsilon}=\partial_{\nu} l_{0 \varepsilon}=0 \quad \text { on } \partial \Omega \quad \text { and } \quad y_{10 \varepsilon}+y_{20 \varepsilon}<1 \quad \text { in } \bar{\Omega} \times[-\tau, 0]
$$

for all $\varepsilon \in(0,1)$ as well as

$$
\left\{\begin{array}{l}
c_{0 \varepsilon} \rightarrow c_{0} \quad \text { in } C^{0}(\bar{\Omega}), \\
v_{0 \varepsilon} \rightarrow v_{0} \quad \text { in } W^{1,2}(\Omega) \cap C^{0}(\bar{\Omega}), \\
l_{0 \varepsilon} \rightarrow l_{0} \quad \text { in } W^{1,2}(\Omega) \cap C^{0}(\bar{\Omega}), \\
\kappa_{0 \varepsilon} \rightarrow \kappa_{0} \quad \text { in } W^{1,4}(\Omega), \\
y_{10 \varepsilon} \rightarrow y_{10} \quad \text { in } C^{0}\left([-\tau, 0] ; W^{1,4}(\Omega)\right) \quad \text { and } \\
y_{20 \varepsilon} \rightarrow y_{20} \quad \text { in } C^{0}\left([-\tau, 0] ; W^{1,4}(\Omega)\right)
\end{array}\right.
$$

as $\varepsilon \searrow 0$.

We shall see in Lemma 3.11 below that each of these problems possesses a global classical solution.

\section{Global existence and basic estimates for the regularized problems}

\subsection{Local existence}

Let us first assert the local existence of smooth solutions to (2.1).

Lemma 3.1 For any $\varepsilon \in(0,1)$ there exist $T_{\varepsilon} \in(0, \infty]$ and a collection of positive functions $c_{\varepsilon}, v_{\varepsilon}, l_{\varepsilon}$, $y_{1 \varepsilon}, y_{2 \varepsilon}$ and $\kappa_{\varepsilon}$, all belonging to $C^{2,1}\left(\bar{\Omega} \times\left[0, T_{\varepsilon}\right)\right)$, which solve (2.1) in the classical sense in $\Omega \times\left(0, T_{\varepsilon}\right)$ and have the additional property that

$$
\text { if } \begin{aligned}
T_{\varepsilon} & <\infty \text { then } \limsup _{t \nearrow T_{\varepsilon}}\left\{\left\|c_{\varepsilon}(\cdot, t)\right\|_{C^{2+\beta}(\bar{\Omega})}+\left\|v_{\varepsilon}(\cdot, t)\right\|_{C^{2+\beta}(\bar{\Omega})}+\left\|l_{\varepsilon}(\cdot, t)\right\|_{C^{2+\beta}(\bar{\Omega})}\right. \\
& \left.+\left\|y_{1 \varepsilon}(\cdot, t)\right\|_{C^{2+\beta}(\bar{\Omega})}+\left\|y_{2 \varepsilon}(\cdot, t)\right\|_{C^{2+\beta}(\bar{\Omega})}+\left\|\kappa_{\varepsilon}(\cdot, t)\right\|_{C^{2+\beta}(\bar{\Omega})}\right\}=\infty \quad \text { for all } \beta \in(0,1) .
\end{aligned}
$$

Proof. We fix $\varepsilon \in(0,1)$ and $\beta \in(0,1)$ and define

$$
\begin{aligned}
A:= & \left\|c_{0 \varepsilon}\right\|_{C^{2+\beta}(\bar{\Omega})}+\left\|v_{0 \varepsilon}\right\|_{C^{2+\beta}(\bar{\Omega})}+\left\|l_{0 \varepsilon}\right\|_{C^{2+\beta}(\bar{\Omega})}+\left\|y_{10 \varepsilon}\right\|_{C^{2+\beta, 1+\frac{\beta}{2}(\bar{\Omega} \times[-\tau, 0])}} \\
& +\left\|y_{20 \varepsilon}\right\|_{C^{2+\beta, 1+\frac{\beta}{2}}(\bar{\Omega} \times[-\tau, 0])}+\left\|\kappa_{0 \varepsilon}\right\|_{C^{2+\beta}(\bar{\Omega})} .
\end{aligned}
$$

Furthermore, we define $c_{0 \varepsilon t}(x)$ to be the right-hand side of the first equation of (2.1) evaluated at $(x, t)=(x, 0)$. This means that

$$
B:=\left\|c_{0 \varepsilon}\right\|_{C^{\beta}(\bar{\Omega})}+\left\|c_{0 \varepsilon t}\right\|_{C^{0}(\bar{\Omega})} \leq C_{1}(A)<\infty
$$


is satisfied with a constant $C_{1}(A)$ depending on $A$. Next, we fix $T \in(0,1]$ and set

$$
X:=\left\{c_{\varepsilon} \in C^{\beta, \frac{\beta}{2}}(\bar{\Omega} \times[0, T]): c_{\varepsilon} \geq 0,\left\|c_{\varepsilon}\right\|_{C^{\beta, \frac{\beta}{2}}(\bar{\Omega} \times[0, T])} \leq B+1\right\} .
$$

Given a fixed $c_{\varepsilon} \in X$, by [16, Theorems V.7.4 and IV.5.3], (2.2), (2.3), $v_{0 \varepsilon}>0$ and the (strong) parabolic maximum principle, there exists a solution $v_{\varepsilon}$ to the second equation of (2.1) with the homogeneous Neumann boundary condition and initial data $v_{0 \varepsilon}$ such that $0<v_{\varepsilon} \leq \max \left\{1,\left\|v_{0 \varepsilon}\right\|_{C^{0}(\bar{\Omega})}\right\}$ in $\bar{\Omega} \times[0, T]$ and

$$
\left\|v_{\varepsilon}\right\|_{C^{2+\beta_{1}, 1+\frac{\beta_{1}}{2}(\bar{\Omega} \times[0, T])}} \leq C_{2}(A)
$$

with some $\beta_{1} \in(0, \beta]$ and some constant $C_{2}(A)>0$ which in view of (3.2) may depend on $A$. Then, using similar arguments we obtain the existence of a positive solution $l_{\varepsilon}$ to the third equation of $(2.1)$ with the homogeneous Neumann boundary condition and initial data $l_{0 \varepsilon}$ satisfying

$$
\left\|l_{\varepsilon}\right\|_{C^{2+\beta_{2}, 1+\frac{\beta_{2}}{2}(\bar{\Omega} \times[0, T])}} \leq C_{3}(A)
$$

with some constant $C_{3}(A)>0$ and some $\beta_{2} \in\left(0, \beta_{1}\right]$. Next, as the fourth and fifth equations of $(2.1)$ form a linear system of ODEs for $\left(y_{1 \varepsilon}, y_{2 \varepsilon}\right)$, by using (3.3), (3.4) and (2.2) we deduce that there exists a solution to this system with initial data $\left(y_{10 \varepsilon}, y_{20 \varepsilon}\right)$ which fulfills $y_{i \varepsilon} \in C^{2+\beta_{2}, 1+\frac{\beta_{2}}{2}}(\bar{\Omega} \times[0, T])$ as well as

$$
\left\|y_{i \varepsilon}\right\|_{C^{\beta_{2}, \frac{\beta_{2}}{2}(\bar{\Omega} \times[-\tau, T-\tau])}}+\left\|\frac{\partial y_{i \varepsilon}}{\partial x_{j}}\right\|_{C^{\beta_{2}, \frac{\beta_{2}}{2}(\bar{\Omega} \times[-\tau, T-\tau])}}+\left\|\frac{\partial^{2} y_{i \varepsilon}}{\partial x_{j} \partial x_{k}}\right\|_{C^{\beta_{2}, \frac{\beta_{2}}{2}}(\bar{\Omega} \times[-\tau, T-\tau])} \leq C_{4}(A)
$$

for all $i \in\{1,2\}$ and $j, k \in\{1, \ldots, n\}$ with some $C_{4}(A)>0$. Since moreover $\bar{Y}$ with $Y:=\left\{\left(y_{1}, y_{2}\right) \in\right.$ $\left.(0,1)^{2}: y_{1}+y_{2}<1\right\}$ is a positive invariant set for this system of ODEs, (2.3), the positivity of the initial data and applications of the comparison principle to $y_{1 \varepsilon}, y_{2 \varepsilon}$ and $y_{1 \varepsilon}+y_{2 \varepsilon}$, respectively, imply that $\left(y_{1 \varepsilon}, y_{2 \varepsilon}\right)$ cannot reach the boundary of $Y$ in finite time and therefore remains inside the positive cone in $\mathbb{R}^{2}$.

Next, in view of $(2.2),(3.5)$ and the positivity of $\kappa_{0 \varepsilon}$, there is a solution $\kappa_{\varepsilon}$ to the linear ODE formed by the sixth equation of (2.1) with initial data $\kappa_{0 \varepsilon}$ which is positive by the comparison principle and satisfies

$$
\left\|\kappa_{\varepsilon}\right\|_{C^{2+\beta_{2}, 1+\frac{\beta_{2}}{2}}(\bar{\Omega} \times[0, T])} \leq C_{5}(A)
$$

with some constant $C_{5}(A)$ depending on $A$. Taking now these functions $v_{\varepsilon}, l_{\varepsilon}$ and $\kappa_{\varepsilon}$ and using [16, Theorems V.7.4 and IV.5.3], (2.2), (2.3), (3.3), (3.4), (3.6) and the positivity of $c_{0 \varepsilon}$, we obtain a solution $\tilde{c}_{\varepsilon} \in C^{2+\beta_{3}, 1+\frac{\beta_{3}}{2}}(\bar{\Omega} \times[0, T])$ to the first equation of (2.1) with the homogeneous Neumann boundary condition and initial data $c_{0 \varepsilon}$ for some $\beta_{3} \in\left(0, \beta_{2}\right]$ which is positive by the strong maximum principle and fulfills

$$
\left\|\tilde{c}_{\varepsilon}\right\|_{C^{2+\beta_{3}, 1+\frac{\beta_{3}}{2}(\bar{\Omega} \times[0, T])}} \leq C_{6}(A)
$$

with some constant $C_{6}(A)$ depending on $A$. In particular, we have

$$
\left\|\partial_{t} \tilde{c}_{\varepsilon}\right\|_{C^{\beta_{3}}, \frac{\beta_{3}}{2}(\bar{\Omega} \times[0, T])} \leq C_{6}(A)
$$


and the regularity of $\tilde{c}_{\varepsilon}$ implies that $c_{0 \varepsilon t}(x)=\partial_{t} \tilde{c}_{\varepsilon}(x, 0)$ for $x \in \bar{\Omega}$ with the definition of $c_{0 \varepsilon t}$ given in the beginning of this proof. Therefore, (3.2) and (3.8) yield the existence of $T_{0} \in(0,1]$ just depending on $A$ such that

$$
\left\|\tilde{c}_{\varepsilon}\right\|_{C^{\beta, \frac{\beta}{2}}\left(\bar{\Omega} \times\left[0, T_{1}\right]\right)} \leq B+1
$$

for $T_{1}:=\min \left\{T_{0}, T\right\}$, where we have used the fact that $\|f\|_{C^{\frac{\beta}{2}\left(\left[0, T_{1}\right]\right)}} \leq\|f\|_{C^{1}\left(\left[0, T_{1}\right]\right)}$ holds for $f \in$ $C^{1}\left(\left[0, T_{1}\right]\right)$ due to $T_{1} \leq 1$. Hence, setting now $T:=T_{0}$, we have $\tilde{c}_{\varepsilon} \in X$ so that the map $F: X \rightarrow X$, $F\left(c_{\varepsilon}\right):=\tilde{c}_{\varepsilon}$ for $c_{\varepsilon} \in X$, is well-defined and compact in view of (3.7). Since $X$ is a closed, bounded and convex subset of $C^{\beta, \frac{\beta}{2}}(\bar{\Omega} \times[0, T]), F$ has a fixed point $c_{\varepsilon}$ by Schauder's fixed point theorem. Starting with this $c_{\varepsilon}$, the above reasoning gives the existence of a classical solution to (2.1) in $\bar{\Omega} \times\left[0, T_{0}\right]$ such that all components are positive and belong to $C^{2,1}\left(\bar{\Omega} \times\left[0, T_{0}\right]\right)$.

Finally, since $T_{0}$ just depends on $A$, the solution to (2.1) can be extended up to some $T_{2}>T_{\varepsilon}$ if all components are uniformly bounded in $C^{2+\beta}(\bar{\Omega})$ for $t \in\left(0, T_{\varepsilon}\right)$ (because in case of $T_{\varepsilon}>\tau>0$ then $y_{1 \varepsilon}$ and $y_{2 \varepsilon}$ are uniformly bounded in $C^{2+\beta, 1+\frac{\beta}{2}}\left(\bar{\Omega} \times\left[0, T_{3}-\tau\right]\right)$ for some $T_{3}>T_{\varepsilon}$ which in view of (3.5) is sufficient to extend the solution up to some $\left.T_{2} \in\left(T_{\varepsilon}, T_{3}\right]\right)$. Hence, $(3.1)$ is proved as $\beta \in(0,1)$ was arbitrary.

\subsection{Some elementary $\varepsilon$-independent estimates}

Some basic but important properties of $c_{\varepsilon}$ and $v_{\varepsilon}$ are summarized in the next two lemmata.

Lemma 3.2 The solution of (2.1) satisfies

$$
\int_{\Omega} c_{\varepsilon}(\cdot, t) \leq m:=\max \left\{\sup _{\varepsilon \in(0,1)} \int_{\Omega} c_{0 \varepsilon},|\Omega|\right\} \quad \text { for all } t \in\left(0, T_{\varepsilon}\right)
$$

and

as well as

$$
\int_{t}^{t+1} \int_{\Omega} c_{\varepsilon}^{2} \leq \frac{\mu_{c}+1}{\mu_{c}} \cdot m \quad \text { for all } t \in\left(0, T_{\varepsilon}-1\right)
$$

$$
\varepsilon \int_{t}^{t+1} \int_{\Omega} c_{\varepsilon}^{\theta} \leq\left(\mu_{c}+1\right) m \quad \text { for all } t \in\left(0, T_{\varepsilon}-1\right) .
$$

Proof. A spatial integration of the first equation in (2.1) yields

$$
\begin{aligned}
\frac{d}{d t} \int_{\Omega} c_{\varepsilon} & =\mu_{c} \int_{\Omega} c_{\varepsilon}\left(1-c_{\varepsilon}-\eta_{1} v_{\varepsilon}\right)-\varepsilon \int_{\Omega} c_{\varepsilon}^{\theta} \\
& \leq \mu_{c} \int_{\Omega} c_{\varepsilon}-\mu_{c} \int_{\Omega} c_{\varepsilon}^{2}-\varepsilon \int_{\Omega} c_{\varepsilon}^{\theta} \quad \text { for all } t \in\left(0, T_{\varepsilon}\right),
\end{aligned}
$$

so that since $\int_{\Omega} c_{\varepsilon}^{2} \geq \frac{1}{|\Omega|}\left(\int_{\Omega} c_{\varepsilon}\right)^{2}$ by the Cauchy-Schwarz inequality, an ODE comparison shows (3.9). Thereafter, (3.10) and (3.11) easily result upon an integration of (3.12) over $(t, t+1)$.

Lemma 3.3 For each $\varepsilon \in(0,1)$, we have

$$
v_{\varepsilon}(x, t) \leq K_{v}:=\max \left\{\sup _{\varepsilon \in(0,1)}\left\|v_{0 \varepsilon}\right\|_{L^{\infty}(\Omega)}, 1\right\} \quad \text { for all } x \in \Omega \text { and } t \in\left(0, T_{\varepsilon}\right) \text {. }
$$


Proof. This is a straightforward consequence of the parabolic maximum principle applied to the second equation in (2.1).

Our next goal is to derive some first regularity properties of $l_{\varepsilon}$. For the argument in the respective Lemma 3.5 below we prepare the following auxiliary statement.

Lemma 3.4 Let $T>0$, and suppose that $z$ is a nonnegative absolutely continuous function on $[0, T)$ satisfying

$$
z^{\prime}(t)+a z(t) \leq f(t) \quad \text { for a.e. } t \in(0, T)
$$

with some $a>0$ and a nonnegative function $f \in L_{l o c}^{1}([0, T))$ for which there exists $b>0$ such that

$$
\int_{t}^{t+1} f(s) d s \leq b \quad \text { for all } t \in[0, T-1) .
$$

Then

$$
z(t) \leq \max \left\{z(0)+b, \frac{b}{a}+2 b\right\} \quad \text { for all } t \in(0, T) .
$$

Proof. In view of the nonnegativity of $z$ and $f$, an integration of (3.13) shows that

$$
z(t) \leq z\left(t_{0}\right)+\int_{t_{0}}^{t} f(s) d s \leq z\left(t_{0}\right)+b \quad \text { for all } t_{0} \in[0, T) \text { and all } t \in\left[t_{0}, t_{0}+1\right] \cap[0, T) .
$$

Next, setting $\gamma:=\max \left\{z(0), \frac{b}{a}+b\right\}$ and $i_{0}:=\max \left\{i \in \mathbb{N}_{0}: i<T\right\}$, we claim that

$$
z(i) \leq \gamma \quad \text { for all } i \in\left\{0, \ldots, i_{0}\right\} .
$$

Obviously, $z(0) \leq \gamma$ is fulfilled. Assuming that $z(i) \leq \gamma$ for some $i \in\left\{0, \ldots, i_{0}-1\right\}$, we either have $z(t) \geq \frac{b}{a}$ for all $t \in[i, i+1]$, so that an integration of (3.13) yields

$$
z(i+1) \leq z(i)-a \cdot \frac{b}{a}+b=z(i) \leq \gamma,
$$

or $z(\tilde{t})<\frac{b}{a}$ for some $\tilde{t} \in[i, i+1]$, which means $z(i+1) \leq z(\tilde{t})+b \leq \gamma$ by (3.15). Hence, (3.16) holds by induction. Finally, a combination of (3.16) and (3.15) with $t_{0}=i$ for $i \in\left\{0, \ldots, i_{0}\right\}$ proves (3.14).

We can now collect a list of estimates for $l_{\varepsilon}$.

Lemma 3.5 There exists $C>0$ such that for all $\varepsilon \in(0,1)$,

$$
\left\|l_{\varepsilon}(\cdot, t)\right\|_{W^{1,2}(\Omega)} \leq C \quad \text { for all } t \in\left(0, T_{\varepsilon}\right)
$$

and

$$
\int_{t}^{t+1}\left\|l_{\varepsilon}(\cdot, t)\right\|_{W^{2,2}(\Omega)}^{2} d s \leq C \quad \text { for all } t \in\left(0, T_{\varepsilon}-1\right)
$$

as well as

$$
\int_{t}^{t+1} \int_{\Omega}\left(\partial_{t} l_{\varepsilon}\right)^{2} \leq C \quad \text { for all } t \in\left(0, T_{\varepsilon}-1\right)
$$

Moreover,

$$
l_{\varepsilon}(x, t) \geq\left\{\inf _{\varepsilon \in(0,1)} \inf _{x \in \Omega} l_{0 \varepsilon}(x)\right\} \cdot e^{-t} \quad \text { for all } x \in \Omega \text { and } t \in\left(0, T_{\varepsilon}\right) .
$$


Proof. We first integrate the third equation in (2.1) and use Lemma 3.2 and Lemma 3.3 to see that

$$
\frac{d}{d t} \int_{\Omega} l_{\varepsilon}=-\int_{\Omega} l_{\varepsilon}+\int_{\Omega} c_{\varepsilon} v_{\varepsilon} \leq-\int_{\Omega} l_{\varepsilon}+m K_{v} \quad \text { for all } t \in\left(0, T_{\varepsilon}\right),
$$

which by an ODE comparison implies that

$$
\int_{\Omega} l_{\varepsilon}(\cdot, t) \leq C_{1}:=\max \left\{\sup _{\varepsilon \in(0,1)} \int_{\Omega} l_{0 \varepsilon}, m K_{v}\right\} \quad \text { for all } t \in\left(0, T_{\varepsilon}\right) .
$$

We next test the PDE for $l_{\varepsilon}$ by $-\Delta l_{\varepsilon}$ and use Young's inequality to find, again relying on Lemma 3.3, that

$$
\begin{aligned}
\frac{1}{2} \frac{d}{d t} \int_{\Omega}\left|\nabla l_{\varepsilon}\right|^{2}+\int_{\Omega}\left|\Delta l_{\varepsilon}\right|^{2}+\int_{\Omega}\left|\nabla l_{\varepsilon}\right|^{2} & =-\int_{\Omega} c_{\varepsilon} v_{\varepsilon} \Delta l_{\varepsilon} \\
& \leq \frac{1}{2} \int_{\Omega}\left|\Delta l_{\varepsilon}\right|^{2}+\frac{K_{v}^{2}}{2} \int_{\Omega} c_{\varepsilon}^{2} \quad \text { for all } t \in\left(0, T_{\varepsilon}\right) .
\end{aligned}
$$

In particular, this shows that $z_{\varepsilon}(t):=\int_{\Omega}\left|\nabla l_{\varepsilon}(\cdot, t)\right|^{2}, t \in\left[0, T_{\varepsilon}\right)$, satisfies

$$
z_{\varepsilon}^{\prime}(t)+2 z_{\varepsilon}(t) \leq f_{\varepsilon}(t):=K_{v}^{2} \int_{\Omega} c_{\varepsilon}^{2}(\cdot, t) \quad \text { for all } t \in\left(0, T_{\varepsilon}\right),
$$

where from Lemma 3.2 we know that

$$
\int_{t}^{t+1} f_{\varepsilon}(s) d s \leq C_{1}:=K_{v}^{2} \cdot \frac{\mu_{c}+1}{\mu_{c}} \cdot m \quad \text { for all } t \in\left[0, T_{\varepsilon}-1\right) .
$$

Hence, Lemma 3.4 applies to warrant that

$$
\int_{\Omega}\left|\nabla l_{\varepsilon}(\cdot, t)\right|^{2} \leq \max \left\{\sup _{\varepsilon \in(0,1)} \int_{\Omega}\left|\nabla l_{0 \varepsilon}\right|^{2}+C_{1}, \frac{5}{2} C_{1}\right\} \quad \text { for all } t \in\left(0, T_{\varepsilon}\right),
$$

which combined with $(3.21)$ and (2.4) yields (3.17).

Now (3.18) easily follows from this and, again, (3.23) and (3.24) upon a time integration in (3.22). The inequality (3.19) is then immediate from (3.18) and (3.10). Finally, (3.20) results upon an application of the parabolic comparison principle.

For the last three components in (2.1) we can derive some pointwise bounds by elementary ODE comparison arguments in the following two lemmata.

Lemma 3.6 Given any $\varepsilon \in(0,1)$, we have the pointwise estimate

$$
y_{1 \varepsilon}(x, t)+y_{2 \varepsilon}(x, t) \leq K_{y}:=1 \quad \text { for all } x \in \Omega \text { and } t \in\left(-\tau, T_{\varepsilon}\right) .
$$

Proof. It is clear by (2.3) that the claimed inequality holds for all $(x, t) \in \Omega \times(-\tau, 0]$. As for positive $t$, we add the respective equations in (2.1) to obtain

$$
\begin{aligned}
\partial_{t}\left(y_{1 \varepsilon}+y_{2 \varepsilon}\right) & =\left\{1-\left(y_{1 \varepsilon}+y_{2 \varepsilon}\right)\right\} \cdot\left\{k_{1} v_{\varepsilon}+k_{2} l_{\varepsilon}\right\}-k_{-1} y_{1 \varepsilon}-k_{-2} y_{2 \varepsilon} \\
& \leq\left\{1-\left(y_{1 \varepsilon}+y_{2 \varepsilon}\right)\right\} \cdot\left\{k_{1} v_{\varepsilon}+k_{2} l_{\varepsilon}\right\} \quad \text { in } \Omega \times\left(0, T_{\varepsilon}\right)
\end{aligned}
$$

which immediately implies (3.25) by an ODE comparison. 
Lemma 3.7 Let $K_{y}$ be as given by Lemma 3.6. Then for all $\varepsilon \in(0,1)$, the function $\kappa_{\varepsilon}$ has the properties

$$
\kappa_{\varepsilon}(x, t) \leq K_{\kappa}:=\max \left\{\sup _{\varepsilon \in(0,1)}\left\|\kappa_{0 \varepsilon}\right\|_{L^{\infty}(\Omega)}, M K_{y}\right\} \quad \text { for all } x \in \Omega \text { and } t \in\left(0, T_{\varepsilon}\right)
$$

and

$$
\kappa_{\varepsilon}(x, t) \geq\left\{\inf _{\varepsilon \in(0,1)} \inf _{x \in \Omega} \kappa_{0 \varepsilon}(x)\right\} \cdot e^{-t} \quad \text { for all } x \in \Omega \text { and } t \in\left(0, T_{\varepsilon}\right)
$$

as well as

$$
\left|\partial_{t} \kappa_{\varepsilon}(x, t)\right| \leq K_{\kappa} \quad \text { for all } x \in \Omega \text { and } t \in\left(0, T_{\varepsilon}\right) .
$$

Proof. $\quad$ Since $0 \leq y_{i \varepsilon}(x, t-\tau) \leq K_{y}$ for all $(x, t) \in \Omega \times\left(0, T_{\varepsilon}\right)$ and $i \in\{1,2\}$ by Lemma 3.6, $\kappa_{\varepsilon}$ satisfies $-\kappa_{\varepsilon} \leq \partial_{t} \kappa_{\varepsilon} \leq-\kappa_{\varepsilon}+M K_{y} \leq M K_{y}$ in $\Omega \times\left(0, T_{\varepsilon}\right)$. By integration, this first implies (3.26) and (3.27) and then proves (3.28) by using (3.26).

\subsection{Global existence in the approximate problems}

We next aim at proving that all problems (2.1) are in fact globally solvable. To this end, in view of (3.1) it is sufficient to derive, given any fixed $\varepsilon \in(0,1)$, bounds for all solution components with respect to the norm in $C^{2+\beta}(\bar{\Omega})$ with some $\beta>0$.

We begin by performing a standard testing procedure to the first equation in (2.1).

Lemma 3.8 Let $\varepsilon \in(0,1)$ and $p>1$. Then there exists $C(\varepsilon, p)>0$ such that

$$
\frac{1}{p} \frac{d}{d t} \int_{\Omega} c_{\varepsilon}^{p}+\frac{(p-1) \varepsilon}{2} \int_{\Omega} c_{\varepsilon}^{p-2}\left|\nabla c_{\varepsilon}\right|^{2} \leq C(\varepsilon, p) \int_{\Omega} c_{\varepsilon}^{p}\left|\nabla v_{\varepsilon}\right|^{2}+C(\varepsilon, p) \int_{\Omega} c_{\varepsilon}^{p}\left|\nabla l_{\varepsilon}\right|^{2}+\mu_{c} \int_{\Omega} c_{\varepsilon}^{p}
$$

for all $t \in\left(0, T_{\varepsilon}\right)$.

Proof. We multiply the first equation in (2.1) by $c_{\varepsilon}^{p-1}$ to see upon integrating by parts and dropping nonnegative terms that

$$
\begin{aligned}
\frac{1}{p} \frac{d}{d t} \int_{\Omega} c_{\varepsilon}^{p}+(p-1) \varepsilon \int_{\Omega} c_{\varepsilon}^{p-2}\left|\nabla c_{\varepsilon}\right|^{2} \leq & (p-1) \int_{\Omega} \frac{\kappa_{\varepsilon} v_{\varepsilon}}{1+v_{\varepsilon}} c_{\varepsilon}^{p-1} \nabla c_{\varepsilon} \cdot \nabla v_{\varepsilon} \\
& +(p-1) \int_{\Omega} \frac{1}{1+c_{\varepsilon} l_{\varepsilon}} c_{\varepsilon}^{p-1} \nabla c_{\varepsilon} \cdot \nabla l_{\varepsilon} \\
& +\mu_{c} \int_{\Omega} c_{\varepsilon}^{p} \quad \text { for all } t \in\left(0, T_{\varepsilon}\right) .
\end{aligned}
$$

Here, recalling Lemma 3.7 and Lemma 3.3 and using Young's inequality we see that

$$
(p-1) \int_{\Omega} \frac{\kappa_{\varepsilon} v_{\varepsilon}}{1+v_{\varepsilon}} c_{\varepsilon}^{p-1} \nabla c_{\varepsilon} \cdot \nabla v_{\varepsilon} \leq \frac{(p-1) \varepsilon}{4} \int_{\Omega} c_{\varepsilon}^{p-2}\left|\nabla c_{\varepsilon}\right|^{2}+\frac{p-1}{\varepsilon} K_{\kappa}^{2} K_{v}^{2} \int_{\Omega} c_{\varepsilon}^{p}\left|\nabla v_{\varepsilon}\right|^{2},
$$

and similarly we obtain

$$
(p-1) \int_{\Omega} \frac{1}{1+c_{\varepsilon} l_{\varepsilon}} c_{\varepsilon}^{p-1} \nabla c_{\varepsilon} \cdot \nabla l_{\varepsilon} \leq \frac{(p-1) \varepsilon}{4} \int_{\Omega} c_{\varepsilon}^{p-2}\left|\nabla c_{\varepsilon}\right|^{2}+\frac{p-1}{\varepsilon} \int_{\Omega} c_{\varepsilon}^{p}\left|\nabla l_{\varepsilon}\right|^{2}
$$


for all $t \in\left(0, T_{\varepsilon}\right)$. Hence, (3.29) results from (3.30).

The next lemma will enable us to estimate the first two integrals on the right of (3.29) suitably. Its proof is the only place where we need our assumption that the exponent in the artificial absorptive term in the first equation of (2.1) satisfies $\theta>\max \{n, 2\}$.

Lemma 3.9 Let $T>0$. Then for each $\varepsilon \in(0,1)$ one can find $C(\varepsilon, T)>0$ such that

$$
\int_{0}^{\widehat{T_{\varepsilon}}}\left(\left\|\nabla v_{\varepsilon}(\cdot, t)\right\|_{L^{\infty}(\Omega)}^{2}+\left\|\nabla l_{\varepsilon}(\cdot, t)\right\|_{L^{\infty}(\Omega)}^{2}\right) d t \leq C(\varepsilon, T)
$$

holds for $\widehat{T}_{\varepsilon}=\min \left\{T, T_{\varepsilon}\right\}$.

Proof. From Lemma 3.2 we know that

$$
\int_{0}^{\widehat{T_{\varepsilon}}} \int_{\Omega} c_{\varepsilon}^{\theta} \leq \frac{\left(\mu_{c}+1\right) m(T+1)}{\varepsilon},
$$

so that since $v_{\varepsilon} \leq K_{v}$ by Lemma $3.3, f_{\varepsilon}:=\partial_{t} v_{\varepsilon}-\varepsilon \Delta v_{\varepsilon} \equiv \mu_{v} v_{\varepsilon}\left(1-v_{\varepsilon}\right)-\lambda c_{\varepsilon} v_{\varepsilon}$ is bounded in $L^{\theta}\left(\Omega \times\left(0, \widehat{T}_{\varepsilon}\right)\right)$. Therefore, known results on maximal Sobolev regularity in parabolic equations ([11]) assert that

$$
\int_{0}^{\widehat{T_{\varepsilon}}}\left\|v_{\varepsilon}(\cdot, t)\right\|_{W^{2, \theta}(\Omega)}^{\theta} d t \leq C_{1}(\varepsilon, T)
$$

with some $C_{1}(\varepsilon, T)>0$. Similarly, also $g_{\varepsilon}:=\partial_{t} l_{\varepsilon}-\Delta l_{\varepsilon}+l_{\varepsilon} \equiv c_{\varepsilon} v_{\varepsilon}$ belongs to $L^{\theta}\left(\Omega \times\left(0, \widehat{T}_{\varepsilon}\right)\right)$, whence applying the same regularity result to the third equation in (2.1) yields $C_{2}(\varepsilon, T)>0$ fulfilling

$$
\int_{0}^{\widehat{T_{\varepsilon}}}\left\|l_{\varepsilon}(\cdot, t)\right\|_{W^{2, \theta}(\Omega)}^{\theta} d t \leq C_{2}(\varepsilon, T) .
$$

As $\theta>2$ and $W^{2, \theta}(\Omega) \hookrightarrow W^{1, \infty}(\Omega)$ thanks to the additional assumption $\theta>n,(3.33)$ and (3.34) imply (3.31).

We can thereby estimate $c_{\varepsilon}$ in $L^{\infty}\left((0, T) ; L^{p}(\Omega)\right)$ for any $p \in(1, \infty)$ and each finite $T \leq T_{\varepsilon}$.

Lemma 3.10 Let $p>1$ and $T>0$. Then for all $\varepsilon \in(0,1)$ there exists $C(\varepsilon, p, T)>0$ such that

$$
\int_{\Omega} c_{\varepsilon}^{p}(\cdot, t) \leq C(\varepsilon, p, T) \quad \text { for all } t \in\left(0, \widehat{T}_{\varepsilon}\right)
$$

Proof. $\quad$ By Lemma 3.8, we can find $C_{1}(\varepsilon, p)>0$ such that

$$
\frac{d}{d t} \int_{\Omega} c_{\varepsilon}^{p} \leq C_{1}(\varepsilon, p) \cdot\left(\left\|\nabla v_{\varepsilon}(\cdot, t)\right\|_{L^{\infty}(\Omega)}^{2}+\left\|\nabla l_{\varepsilon}(\cdot, t)\right\|_{L^{\infty}(\Omega)}^{2}\right) \cdot \int_{\Omega} c_{\varepsilon}^{p}+p \mu_{c} \int_{\Omega} c_{\varepsilon}^{p}
$$

for all $t \in\left(0, T_{\varepsilon}\right)$, so that an integration yields

$$
\int_{\Omega} c_{\varepsilon}^{p}(\cdot, t) \leq\left(\int_{\Omega} c_{0 \varepsilon}^{p}\right) \cdot \exp \left\{C_{1}(\varepsilon, p) \cdot \int_{0}^{t}\left(\left\|\nabla v_{\varepsilon}(\cdot, s)\right\|_{L^{\infty}(\Omega)}^{2}+\left\|\nabla l_{\varepsilon}(\cdot, s)\right\|_{L^{\infty}(\Omega)}^{2}\right) d s+p \mu_{c} t\right\}
$$

for all $t \in\left(0, T_{\varepsilon}\right)$. In view of Lemma 3.9, this proves (3.35).

Now the desired result on global existence in (2.1) can be derived in a straightforward manner. 
Lemma 3.11 For each $\varepsilon \in(0,1)$, the solution of (2.1) from Lemma 3.1 is global in time; that is, we have $T_{\varepsilon}=\infty$.

Proof. Assuming on the contrary that $T_{\varepsilon}$ is finite for some $\varepsilon \in(0,1)$, from Lemma 3.10 and parabolic regularity theory ([16]) applied to the second and third equation in (2.1) in combination with Lemma 3.3 and Lemma 3.7 we would obtain that the coefficient functions

$$
\begin{aligned}
a_{\varepsilon}(x, t, q):= & \varepsilon q+\frac{\kappa_{\varepsilon}(x, t)}{1+c_{\varepsilon}(x, t) v_{\varepsilon}(x, t)} q-\frac{\kappa_{\varepsilon}(x, t) v_{\varepsilon}(x, t)}{1+v_{\varepsilon}(x, t)} c_{\varepsilon}(x, t) \nabla v_{\varepsilon}(x, t) \\
& -\frac{c_{\varepsilon}(x, t)}{1+c_{\varepsilon}(x, t) l_{\varepsilon}(x, t)} \nabla l_{\varepsilon}(x, t), \quad(x, t, q) \in \Omega \times\left(0, T_{\varepsilon}\right) \times \mathbb{R}^{n},
\end{aligned}
$$

and

$$
b_{\varepsilon}(x, t):=\mu_{c} c_{\varepsilon}(x, t) \cdot\left(1-c_{\varepsilon}(x, t)-\eta_{1} v_{\varepsilon}(x, t)\right)-\varepsilon c_{\varepsilon}^{\theta}(x, t), \quad(x, t) \in \Omega \times\left(0, T_{\varepsilon}\right),
$$

in

$$
\partial_{t} c_{\varepsilon}=\nabla \cdot\left(a_{\varepsilon}\left(x, t, \nabla c_{\varepsilon}\right)\right)+b_{\varepsilon}(x, t), \quad(x, t) \in \Omega \times\left(0, T_{\varepsilon}\right),
$$

would satisfy

$$
a_{\varepsilon}(x, t, q) q \geq \frac{\varepsilon}{2}|q|^{2}-\psi_{0}(x, t) \quad \text { for all }(x, t, q) \in \Omega \times\left(0, T_{\varepsilon}\right) \times \mathbb{R}^{n}
$$

and

$$
\left|a_{\varepsilon}(x, t, q)\right| \leq\left(\varepsilon+K_{\kappa}\right)|q|+\psi_{1}(x, t) \quad \text { for all }(x, t, q) \in \Omega \times\left(0, T_{\varepsilon}\right) \times \mathbb{R}^{n}
$$

as well as

$$
\left|b_{\varepsilon}(x, t)\right| \leq \psi_{2}(x, t) \quad \text { for all }(x, t) \in \Omega \times\left(0, T_{\varepsilon}\right)
$$

with certain functions $\psi_{0}, \psi_{1}$ and $\psi_{2}$ all belonging to $\bigcap_{p>1} L^{p}\left(\Omega \times\left(0, T_{\varepsilon}\right)\right)$. Therefore, parabolic Hölder estimates ([26, Theorem 1.3 and Remark 1.4]) would yield $\beta \in(0,1)$ and $C_{1}(\varepsilon)>0$ such that

$$
\left\|c_{\varepsilon}\right\|_{C^{\beta, \frac{\beta}{2}}\left(\bar{\Omega} \times\left[0, T_{\varepsilon}\right]\right)} \leq C_{1}(\varepsilon) .
$$

According to standard parabolic Schauder estimates $([16])$, this in turn would provide bounds for $v_{\varepsilon}$ and $l_{\varepsilon}$ in $C^{2+\beta, 1+\frac{\beta}{2}}\left(\bar{\Omega} \times\left[0, T_{\varepsilon}\right]\right)$, which would directly entail corresponding estimates for $y_{1 \varepsilon}, y_{2 \varepsilon}$ and $\kappa_{\varepsilon}$ in the latter space. Again by parabolic Schauder theory, this would now imply that also $c_{\varepsilon}$ lies in $C^{2+\beta, 1+\frac{\beta}{2}}\left(\bar{\Omega} \times\left[0, T_{\varepsilon}\right]\right)$ and thereby contradict the extensibility criterion (3.1) in Lemma 3.1.

\section{An entropy-type functional}

The goal of the present section is to establish the following estimate which has its origin in (1.9). 
Lemma 4.1 Let $T>0$. Then there exists $C(T)>0$ such that for any choice of $\varepsilon \in(0,1)$ the solution of (2.1) satisfies

$$
\begin{aligned}
\sup _{t \in(0, T)}\left\{\int_{\Omega} c_{\varepsilon} \ln c_{\varepsilon}+\int_{\Omega} \frac{\kappa_{\varepsilon}\left|\nabla v_{\varepsilon}\right|^{2}}{1+v_{\varepsilon}}\right\} & +\varepsilon \int_{0}^{T} \int_{\Omega} \frac{\left|\nabla c_{\varepsilon}\right|^{2}}{c_{\varepsilon}}+\int_{0}^{T} \int_{\Omega} \frac{\kappa_{\varepsilon}}{1+c_{\varepsilon} v_{\varepsilon}} \frac{\left|\nabla c_{\varepsilon}\right|^{2}}{c_{\varepsilon}} \\
& +\int_{0}^{T} \int_{\Omega} \kappa_{\varepsilon} c_{\varepsilon} \frac{\left|\nabla v_{\varepsilon}\right|^{2}}{\left(1+v_{\varepsilon}\right)^{2}}+\int_{0}^{T} \int_{\Omega} c_{\varepsilon}^{2} \ln \left(2+c_{\varepsilon}\right) \\
& +\varepsilon \int_{0}^{T} \int_{\Omega} c_{\varepsilon}^{\theta} \ln \left(2+c_{\varepsilon}\right) \\
\leq & C(T) .
\end{aligned}
$$

The proof thereof will be prepared by a series of integral estimates. The first of these, to be given in Lemma 4.3, requires itself as a preliminary the following elementary observation.

Lemma 4.2 There exists $C>0$ such that

$$
\xi \ln \xi-\xi^{2} \ln \xi \leq-\frac{1}{2} \xi^{2} \ln (2+\xi)+C \quad \text { for all } \xi>0
$$

and

$$
-\xi^{\theta} \ln \xi \leq-\frac{1}{2} \xi^{\theta} \ln (2+\xi)+C \quad \text { for all } \xi>0 \text { and } \theta>0 .
$$

Proof. To see (4.2), we note that $\phi:[0, \infty) \rightarrow \mathbb{R}$ with $\phi(0):=0$ and $\phi(\xi):=\xi \ln \xi-\xi^{2} \ln \xi+$ $\frac{1}{2} \xi^{2} \ln (2+\xi), \xi>0$, defines a continuous function which satisfies $\phi(\xi) \rightarrow-\infty$ as $\xi \rightarrow \infty$, so that $\phi(\xi)<0$ for all $\xi>\xi_{0}$ with some $\xi_{0}>0$. Thus, (4.2) follows if we pick any $C>\max _{\xi \in\left[0, \xi_{0}\right]} \phi(\xi)$. A verification of (4.3) can be achieved in much the same manner.

We can thereby give some basic information on the time evolution of the first integral on the left of (4.1).

Lemma 4.3 There exists $C>0$ such that for any $\varepsilon \in(0,1)$ we have

$$
\begin{aligned}
\frac{d}{d t} \int_{\Omega} c_{\varepsilon} \ln c_{\varepsilon} & +\varepsilon \int_{\Omega} \frac{\left|\nabla c_{\varepsilon}\right|^{2}}{c_{\varepsilon}}+\frac{1}{2} \int_{\Omega} \frac{\kappa_{\varepsilon}}{1+c_{\varepsilon} v_{\varepsilon}} \frac{\left|\nabla c_{\varepsilon}\right|^{2}}{c_{\varepsilon}}+\frac{\mu_{c}}{2} \int_{\Omega} c_{\varepsilon}^{2} \ln \left(2+c_{\varepsilon}\right)+\frac{\varepsilon}{2} \int_{\Omega} c_{\varepsilon}^{\theta} \ln \left(2+c_{\varepsilon}\right) \\
& \leq \int_{\Omega} \frac{\kappa_{\varepsilon} v_{\varepsilon}}{1+v_{\varepsilon}} \nabla c_{\varepsilon} \cdot \nabla v_{\varepsilon}+\frac{1}{2} \int_{\Omega} \frac{c_{\varepsilon}\left(1+c_{\varepsilon} v_{\varepsilon}\right)}{\kappa_{\varepsilon}\left(1+c_{\varepsilon} l_{\varepsilon}\right)^{2}}\left|\nabla l_{\varepsilon}\right|^{2}+C \quad \text { for all } t>0 .
\end{aligned}
$$

ProOF. Since $c_{\varepsilon}$ is positive in $\bar{\Omega} \times[0, \infty)$ according to the strong maximum principle, we can use the first equation in (2.1) to compute

$$
\begin{aligned}
\frac{d}{d t} \int_{\Omega} c_{\varepsilon} \ln c_{\varepsilon}= & \int_{\Omega} \ln c_{\varepsilon} \cdot \partial_{t} c_{\varepsilon}+\int_{\Omega} \partial_{t} c_{\varepsilon} \\
= & -\varepsilon \int_{\Omega} \frac{\left|\nabla c_{\varepsilon}\right|^{2}}{c_{\varepsilon}}-\int_{\Omega} \frac{\kappa_{\varepsilon}}{1+c_{\varepsilon} v_{\varepsilon}} \frac{\left|\nabla c_{\varepsilon}\right|^{2}}{c_{\varepsilon}}+\int_{\Omega} \frac{\kappa_{\varepsilon} v_{\varepsilon}}{1+v_{\varepsilon}} \nabla c_{\varepsilon} \cdot \nabla v_{\varepsilon}+\int_{\Omega} \frac{1}{1+c_{\varepsilon} l_{\varepsilon}} \nabla c_{\varepsilon} \cdot \nabla l_{\varepsilon} \\
& +\mu_{c} \int_{\Omega}\left\{c_{\varepsilon} \ln c_{\varepsilon}-c_{\varepsilon}^{2} \ln c_{\varepsilon}\right\}-\mu_{c} \eta_{1} \int_{\Omega} c_{\varepsilon} \ln c_{\varepsilon} v_{\varepsilon}-\varepsilon \int_{\Omega} c_{\varepsilon}^{\theta} \ln c_{\varepsilon} \\
& +\mu_{c} \int_{\Omega} c_{\varepsilon}\left(1-c_{\varepsilon}-\eta_{1} v_{\varepsilon}\right)-\varepsilon \int_{\Omega} c_{\varepsilon}^{\theta} \quad \text { for all } t>0
\end{aligned}
$$


where by Young's inequality

$$
\int_{\Omega} \frac{1}{1+c_{\varepsilon} l_{\varepsilon}} \nabla c_{\varepsilon} \cdot \nabla l_{\varepsilon} \leq \frac{1}{2} \int_{\Omega} \frac{\kappa_{\varepsilon}}{1+c_{\varepsilon} v_{\varepsilon}} \frac{\left|\nabla c_{\varepsilon}\right|^{2}}{c_{\varepsilon}}+\frac{1}{2} \int_{\Omega} \frac{c_{\varepsilon}\left(1+c_{\varepsilon} v_{\varepsilon}\right)}{\kappa_{\varepsilon}\left(1+c_{\varepsilon} l_{\varepsilon}\right)^{2}}\left|\nabla l_{\varepsilon}\right|^{2} .
$$

Moreover, from Lemma 4.2 we obtain $C_{1}>0$ and $C_{2}>0$ such that

$$
\mu_{c} \int_{\Omega}\left\{c_{\varepsilon} \ln c_{\varepsilon}-c_{\varepsilon}^{2} \ln c_{\varepsilon}\right\} \leq-\frac{\mu_{c}}{2} \int_{\Omega} c_{\varepsilon}^{2} \ln \left(2+c_{\varepsilon}\right)+C_{1}
$$

and

$$
-\varepsilon \int_{\Omega} c_{\varepsilon}^{\theta} \ln c_{\varepsilon} \leq-\frac{\varepsilon}{2} \int_{\Omega} c_{\varepsilon}^{\theta} \ln \left(2+c_{\varepsilon}\right)+C_{2}
$$

whereas clearly

$$
\mu_{c} \int_{\Omega} c_{\varepsilon}\left(1-c_{\varepsilon}-\eta_{1} v_{\varepsilon}\right)-\varepsilon \int_{\Omega} c_{\varepsilon}^{\theta} \leq \mu_{c}|\Omega|
$$

and, with $K_{v}$ as in Lemma 3.3,

$$
-\mu_{c} \eta_{1} \int_{\Omega} c_{\varepsilon} \ln c_{\varepsilon} v_{\varepsilon} \leq \mu_{c} \eta_{1} \frac{K_{v}|\Omega|}{e}
$$

the latter because $\xi \ln \xi \geq-\frac{1}{e}$ for all $\xi>0$. Combining (4.5)-(4.10) directly leads to (4.4).

Next, the integrand in the second term on the left of (4.1) even satisfies a favorable pointwise inequality. We note that all terms appearing below are meaningful since $v_{\varepsilon}$ is smooth in $\bar{\Omega} \times\left(0, T_{\varepsilon}\right)$ by standard parabolic regularity theory ([16]).

Lemma 4.4 Let $K_{\kappa}$ be as given by Lemma 3.7. Then for all $\varepsilon \in(0,1)$ we have the pointwise inequality

$$
\begin{aligned}
\partial_{t} \frac{\kappa_{\varepsilon}\left|\nabla v_{\varepsilon}\right|^{2}}{1+v_{\varepsilon}} \leq & 2 \varepsilon \cdot \frac{\kappa_{\varepsilon}}{1+v_{\varepsilon}} \nabla v_{\varepsilon} \cdot \nabla \Delta v_{\varepsilon}-\varepsilon \frac{\kappa_{\varepsilon}}{\left(1+v_{\varepsilon}\right)^{2}}\left|\nabla v_{\varepsilon}\right|^{2} \Delta v_{\varepsilon} \\
& -2 \lambda \cdot \frac{\kappa_{\varepsilon} v_{\varepsilon}}{1+v_{\varepsilon}} \nabla c_{\varepsilon} \cdot \nabla v_{\varepsilon}-2 \lambda \kappa_{\varepsilon} c_{\varepsilon} \frac{\left|\nabla v_{\varepsilon}\right|^{2}}{\left(1+v_{\varepsilon}\right)^{2}} \\
& +\left(2 \mu_{v}+1\right) K_{\kappa} \frac{\left|\nabla v_{\varepsilon}\right|^{2}}{1+v_{\varepsilon}} \quad \text { for all } x \in \Omega \text { and } t>0 .
\end{aligned}
$$

Proof. By straightforward differentiation we obtain

$$
\partial_{t} \frac{\kappa_{\varepsilon}\left|\nabla v_{\varepsilon}\right|^{2}}{1+v_{\varepsilon}}=\left\{\frac{2 \kappa_{\varepsilon} \nabla v_{\varepsilon} \cdot \nabla\left(\partial_{t} v_{\varepsilon}\right)}{1+v_{\varepsilon}}-\frac{\kappa_{\varepsilon}\left|\nabla v_{\varepsilon}\right|^{2} \partial_{t} v_{\varepsilon}}{\left(1+v_{\varepsilon}\right)^{2}}\right\}+\frac{\partial_{t} \kappa_{\varepsilon}\left|\nabla v_{\varepsilon}\right|^{2}}{1+v_{\varepsilon}} \quad \text { for all } x \in \Omega \text { and } t>0
$$

where using the second equation in (2.1) in its differentiated version,

$$
\nabla\left(\partial_{t} v_{\varepsilon}\right)=\varepsilon \nabla \Delta v_{\varepsilon}+\mu_{v} \nabla v_{\varepsilon}-2 \mu_{v} v_{\varepsilon} \nabla v_{\varepsilon}-\lambda c_{\varepsilon} \nabla v_{\varepsilon}-\lambda v_{\varepsilon} \nabla c_{\varepsilon},
$$


we compute

$$
\begin{aligned}
\frac{2 \kappa_{\varepsilon} \nabla v_{\varepsilon} \cdot \nabla\left(\partial_{t} v_{\varepsilon}\right)}{1+}-v_{\varepsilon} & \frac{\kappa_{\varepsilon}\left|\nabla v_{\varepsilon}\right|^{2} \partial_{t} v_{\varepsilon}}{\left(1+v_{\varepsilon}\right)^{2}} \\
= & \kappa_{\varepsilon} \cdot\left\{2 \varepsilon \frac{1}{1+v_{\varepsilon}} \nabla v_{\varepsilon} \cdot \nabla \Delta v_{\varepsilon}+2 \mu_{v} \frac{\left|\nabla v_{\varepsilon}\right|^{2}}{1+v_{\varepsilon}}-4 \mu_{v} \frac{v_{\varepsilon}\left|\nabla v_{\varepsilon}\right|^{2}}{1+v_{\varepsilon}}-2 \lambda \frac{c_{\varepsilon}\left|\nabla v_{\varepsilon}\right|^{2}}{1+v_{\varepsilon}}\right. \\
& \quad-2 \lambda \frac{v_{\varepsilon}}{1+v_{\varepsilon}} \nabla c_{\varepsilon} \cdot \nabla v_{\varepsilon}-\varepsilon \frac{1}{\left(1+v_{\varepsilon}\right)^{2}}\left|\nabla v_{\varepsilon}\right|^{2} \Delta v_{\varepsilon}-\mu_{v} \frac{v_{\varepsilon}\left|\nabla v_{\varepsilon}\right|^{2}}{\left(1+v_{\varepsilon}\right)^{2}}+\mu_{v} \frac{v_{\varepsilon}^{2}\left|\nabla v_{\varepsilon}\right|^{2}}{\left(1+v_{\varepsilon}\right)^{2}} \\
& \left.\quad+\lambda \frac{c_{\varepsilon} v_{\varepsilon}\left|\nabla v_{\varepsilon}\right|^{2}}{\left(1+v_{\varepsilon}\right)^{2}}\right\} \\
= & 2 \varepsilon \frac{\kappa_{\varepsilon}}{1+v_{\varepsilon}} \nabla v_{\varepsilon} \cdot \nabla \Delta v_{\varepsilon}-\varepsilon \frac{\kappa_{\varepsilon}}{\left(1+v_{\varepsilon}\right)^{2}}\left|\nabla v_{\varepsilon}\right|^{2} \Delta v_{\varepsilon}-2 \lambda \frac{\kappa_{\varepsilon} v_{\varepsilon}}{1+v_{\varepsilon}} \nabla c_{\varepsilon} \cdot \nabla v_{\varepsilon} \\
& +\frac{\kappa_{\varepsilon}\left|\nabla v_{\varepsilon}\right|^{2}}{\left(1+v_{\varepsilon}\right)^{2}} \cdot\left\{2 \mu_{v}\left(1+v_{\varepsilon}\right)-4 \mu_{v} v_{\varepsilon}\left(1+v_{\varepsilon}\right)-2 \lambda c_{\varepsilon}\left(1+v_{\varepsilon}\right)-\mu_{v} v_{\varepsilon}+\mu_{v} v_{\varepsilon}^{2}+\lambda c_{\varepsilon} v_{\varepsilon}\right\} \\
= & 2 \varepsilon \frac{\kappa_{\varepsilon}}{1+v_{\varepsilon}} \nabla v_{\varepsilon} \cdot \nabla \Delta v_{\varepsilon}-\varepsilon \frac{\kappa_{\varepsilon}}{\left(1+v_{\varepsilon}\right)^{2}}\left|\nabla v_{\varepsilon}\right|^{2} \Delta v_{\varepsilon}-2 \lambda \frac{\kappa_{\varepsilon} v_{\varepsilon}}{1+v_{\varepsilon}} \nabla c_{\varepsilon} \cdot \nabla v_{\varepsilon} \\
& +\frac{\kappa_{\varepsilon}\left|\nabla v_{\varepsilon}\right|^{2}}{\left(1+v_{\varepsilon}\right)^{2}} \cdot\left\{2 \mu_{v}-3 \mu_{v} v_{\varepsilon}-3 \mu_{v} v_{\varepsilon}^{2}-2 \lambda c_{\varepsilon}-\lambda c_{\varepsilon} v_{\varepsilon}\right\} \\
\leq & 2 \varepsilon \frac{\kappa_{\varepsilon}}{1+v_{\varepsilon}} \nabla v_{\varepsilon} \cdot \nabla \Delta v_{\varepsilon}-\varepsilon \frac{\kappa_{\varepsilon}}{\left(1+v_{\varepsilon}\right)^{2}}\left|\nabla v_{\varepsilon}\right|^{2} \Delta v_{\varepsilon}-2 \lambda \frac{\kappa_{\varepsilon} v_{\varepsilon}}{1+v_{\varepsilon}} \nabla c_{\varepsilon} \cdot \nabla v_{\varepsilon} \\
& +2 \mu_{v} \frac{\kappa_{\varepsilon}\left|\nabla v_{\varepsilon}\right|^{2}}{\left(1+v_{\varepsilon}\right)^{2}}-2 \lambda \kappa_{\varepsilon} c_{\varepsilon} \frac{\left|\nabla v_{\varepsilon}\right|^{2}}{\left(1+v_{\varepsilon}\right)^{2}}
\end{aligned}
$$

for all $(x, t) \in \Omega \times(0, \infty)$. Since Lemma 3.7 says that $\kappa_{\varepsilon} \leq K_{\kappa}$ and $\partial_{t} \kappa_{\varepsilon} \leq K_{\kappa}$ in $\Omega \times(0, \infty)$, using that $v_{\varepsilon} \geq 0$ we can estimate

$$
2 \mu_{v} \frac{\kappa_{\varepsilon}\left|\nabla v_{\varepsilon}\right|^{2}}{\left(1+v_{\varepsilon}\right)^{2}}+\frac{\partial_{t} \kappa_{\varepsilon}\left|\nabla v_{\varepsilon}\right|^{2}}{1+v_{\varepsilon}} \leq\left(2 \mu_{v} K_{\kappa}+K_{\kappa}\right) \frac{\left|\nabla v_{\varepsilon}\right|^{2}}{1+v_{\varepsilon}} \quad \text { in } \Omega \times(0, \infty),
$$

and thereby conclude from (4.12) that indeed (4.11) is valid.

During our spatial integration of the above inequality (4.11), a crucial role will be played by the following result of a straightforward computation.

Lemma 4.5 For each $\varepsilon \in(0,1)$ we have

$$
\begin{aligned}
& 2 \varepsilon \int_{\Omega} \frac{\kappa_{\varepsilon}}{1+v_{\varepsilon}} \nabla v_{\varepsilon} \cdot \nabla \Delta v_{\varepsilon}-\varepsilon \int_{\Omega} \frac{\kappa_{\varepsilon}}{\left(1+v_{\varepsilon}\right)^{2}}\left|\nabla v_{\varepsilon}\right|^{2} \Delta v_{\varepsilon} \\
& =-2 \varepsilon \int_{\Omega} \kappa_{\varepsilon}\left(1+v_{\varepsilon}\right)\left|D^{2} \ln \left(1+v_{\varepsilon}\right)\right|^{2}-2 \varepsilon \int_{\Omega} \frac{1}{1+v_{\varepsilon}} \nabla \kappa_{\varepsilon} \cdot\left(D^{2} v_{\varepsilon} \cdot \nabla v_{\varepsilon}\right) \\
& \quad+\varepsilon \int_{\Omega} \frac{1}{\left(1+v_{\varepsilon}\right)^{2}}\left|\nabla v_{\varepsilon}\right|^{2} \nabla \kappa_{\varepsilon} \cdot \nabla v_{\varepsilon}
\end{aligned}
$$

for all $t>0$. 
Proof. Using that $\partial_{\nu} v_{\varepsilon}=0$ on $\partial \Omega$, we may integrate by parts to see that

$$
\begin{aligned}
2 \int_{\Omega} \frac{\kappa_{\varepsilon}}{1+v_{\varepsilon}} \nabla v_{\varepsilon} \cdot \nabla \Delta v_{\varepsilon}= & 2 \sum_{i, j=1}^{n} \int_{\Omega} \frac{\kappa_{\varepsilon}}{1+v_{\varepsilon}} \partial_{j} v_{\varepsilon} \partial_{i i j} v_{\varepsilon} \\
= & -2 \sum_{i, j=1}^{n} \int_{\Omega} \frac{\kappa_{\varepsilon}}{1+v_{\varepsilon}}\left(\partial_{i j} v_{\varepsilon}\right)^{2}+2 \sum_{i, j=1}^{n} \int_{\Omega} \frac{\kappa_{\varepsilon}}{\left(1+v_{\varepsilon}\right)^{2}} \partial_{i} v_{\varepsilon} \partial_{j} v_{\varepsilon} \partial_{i j} v_{\varepsilon} \\
& -2 \sum_{i, j=1}^{n} \int_{\Omega} \frac{\partial_{i} \kappa_{\varepsilon}}{1+v_{\varepsilon}} \partial_{j} v_{\varepsilon} \partial_{i j} v_{\varepsilon}
\end{aligned}
$$

as well as

$$
\begin{aligned}
-\int_{\Omega} \frac{\kappa_{\varepsilon}}{\left(1+v_{\varepsilon}\right)^{2}}\left|\nabla v_{\varepsilon}\right|^{2} \Delta v_{\varepsilon}= & -\sum_{i, j=1}^{n} \int_{\Omega} \frac{\kappa_{\varepsilon}}{\left(1+v_{\varepsilon}\right)^{2}}\left(\partial_{j} v_{\varepsilon}\right)^{2} \partial_{i i} v_{\varepsilon} \\
= & 2 \sum_{i, j=1}^{n} \int_{\Omega} \frac{\kappa_{\varepsilon}}{\left(1+v_{\varepsilon}\right)^{2}} \partial_{i} v_{\varepsilon} \partial_{j} v_{\varepsilon} \partial_{i j} v_{\varepsilon}-2 \sum_{i, j=1}^{n} \int_{\Omega} \frac{\kappa_{\varepsilon}}{\left(1+v_{\varepsilon}\right)^{3}}\left(\partial_{i} v_{\varepsilon}\right)^{2}\left(\partial_{j} v_{\varepsilon}\right)^{2} \\
& +\sum_{i, j=1}^{n} \int_{\Omega} \frac{\partial_{i} \kappa_{\varepsilon}}{\left(1+v_{\varepsilon}\right)^{2}} \partial_{i} v_{\varepsilon}\left(\partial_{j} v_{\varepsilon}\right)^{2}
\end{aligned}
$$

for all $t>0$. Adding both identities yields

$$
\begin{aligned}
& 2 \int_{\Omega} \frac{\kappa_{\varepsilon}}{1+v_{\varepsilon}} \nabla v_{\varepsilon} \cdot \nabla \Delta v_{\varepsilon}-\int_{\Omega} \frac{\kappa_{\varepsilon}}{\left(1+v_{\varepsilon}\right)^{2}}\left|\nabla v_{\varepsilon}\right|^{2} \Delta v_{\varepsilon} \\
&=-2 \sum_{i, j=1}^{n} \int_{\Omega} \kappa_{\varepsilon}\left\{\frac{\left(\partial_{i j} v_{\varepsilon}\right)^{2}}{1+v_{\varepsilon}}-2 \frac{\partial_{i} v_{\varepsilon} \partial_{j} v_{\varepsilon} \partial_{i j} v_{\varepsilon}}{\left(1+v_{\varepsilon}\right)^{2}}+\frac{\left(\partial_{i} v_{\varepsilon}\right)^{2}\left(\partial_{j} v_{\varepsilon}\right)^{2}}{\left(1+v_{\varepsilon}\right)^{3}}\right\} \\
&-2 \int_{\Omega} \frac{1}{1+v_{\varepsilon}} \nabla \kappa_{\varepsilon} \cdot\left(D^{2} v_{\varepsilon} \cdot \nabla v_{\varepsilon}\right)+\int_{\Omega} \frac{1}{\left(1+v_{\varepsilon}\right)^{2}}\left|\nabla v_{\varepsilon}\right|^{2} \nabla \kappa_{\varepsilon} \cdot \nabla v_{\varepsilon} \\
&=-2 \sum_{i, j=1}^{n} \int_{\Omega} \frac{\kappa_{\varepsilon}}{1+v_{\varepsilon}} \cdot\left|\partial_{i j} v_{\varepsilon}-\frac{\partial_{i} v_{\varepsilon} \partial_{j} v_{\varepsilon}}{1+v_{\varepsilon}}\right|^{2} \\
&-2 \int_{\Omega} \frac{1}{1+v_{\varepsilon}} \nabla \kappa_{\varepsilon} \cdot\left(D^{2} v_{\varepsilon} \cdot \nabla v_{\varepsilon}\right)+\int_{\Omega} \frac{1}{\left(1+v_{\varepsilon}\right)^{2}}\left|\nabla v_{\varepsilon}\right|^{2} \nabla \kappa_{\varepsilon} \cdot \nabla v_{\varepsilon}
\end{aligned}
$$

for all $t>0$. As on the other hand we have the pointwise identity

$$
\begin{aligned}
\left(1+v_{\varepsilon}\right)\left|D^{2} \ln \left(1+v_{\varepsilon}\right)\right|^{2} & =\left(1+v_{\varepsilon}\right) \sum_{i, j=1}^{n}\left|\partial_{i}\left\{\frac{\partial_{j} v_{\varepsilon}}{1+v_{\varepsilon}}\right\}\right|^{2} \\
& =\left(1+v_{\varepsilon}\right) \sum_{i, j=1}^{n}\left|\frac{\partial_{i j} v_{\varepsilon}}{1+v_{\varepsilon}}-\frac{\partial_{i} v_{\varepsilon} \partial_{j} v_{\varepsilon}}{\left(1+v_{\varepsilon}\right)^{2}}\right|^{2} \\
& =\sum_{i, j=1}^{n} \frac{1}{1+v_{\varepsilon}} \cdot\left|\partial_{i j} v_{\varepsilon}-\frac{\partial_{i} v_{\varepsilon} \partial_{j} v_{\varepsilon}}{1+v_{\varepsilon}}\right|^{2},
\end{aligned}
$$


from (4.14) we immediately obtain (4.13).

In estimating those integrals in (4.13) which involve $\nabla \kappa_{\varepsilon}$, we shall make use of the following result, which is a special case of a more general integral inequality in [42, Lemma 3.3].

Lemma 4.6 For each nonnegative $\psi \in C^{2}(\bar{\Omega})$, the inequality

$$
\int_{\Omega} \frac{|\nabla \psi|^{4}}{(1+\psi)^{3}} \leq(2+\sqrt{n})^{2} \int_{\Omega}(1+\psi)\left|D^{2} \ln (1+\psi)\right|^{2}
$$

is valid.

Now it is evident that even with this lemma at hand we will not be able to completely suppress integrals involving $\nabla \kappa_{\varepsilon}$. In view of Lemma 4.4 and Lemma 4.5, these will appear along with factors of order $\varepsilon$; more precisely, it will turn out (cf. the proof of Lemma 4.1 below) that we need to control $\varepsilon \int_{\Omega}\left|\nabla \kappa_{\varepsilon}\right|^{4}$ appropriately.

Lemma 4.7 One can find $C>0$ such that for any $\varepsilon \in(0,1)$ we have

$$
\frac{d}{d t} \int_{\Omega}\left|\nabla \kappa_{\varepsilon}\right|^{4} \leq C \cdot\left\{\int_{\Omega}\left|\nabla y_{1 \varepsilon}(\cdot, t-\tau)\right|^{4}+\int_{\Omega}\left|\nabla y_{2 \varepsilon}(\cdot, t-\tau)\right|^{4}\right\} \quad \text { for all } t>0 .
$$

Proof. We compute

$\nabla\left(\partial_{t} \kappa_{\varepsilon}\right)=-\nabla \kappa_{\varepsilon}+\frac{M}{1+y_{2 \varepsilon}(\cdot, t-\tau)} \nabla y_{1 \varepsilon}(\cdot, t-\tau)-\frac{M y_{1 \varepsilon}(\cdot, t-\tau)}{\left(1+y_{2 \varepsilon}(\cdot, t-\tau)\right)^{2}} \nabla y_{2 \varepsilon}(\cdot, t-\tau), \quad x \in \Omega, t>0$, and thus infer using Young's inequality that

$$
\begin{aligned}
\frac{1}{4} \frac{d}{d t} \int_{\Omega}\left|\nabla \kappa_{\varepsilon}\right|^{4}= & -\int_{\Omega}\left|\nabla \kappa_{\varepsilon}\right|^{4}+M \int_{\Omega} \frac{1}{1+y_{2 \varepsilon}(\cdot, t-\tau)}\left|\nabla \kappa_{\varepsilon}\right|^{2} \nabla \kappa_{\varepsilon} \cdot \nabla y_{1 \varepsilon}(\cdot, t-\tau) \\
& -M \int_{\Omega} \frac{y_{1 \varepsilon}(\cdot, t-\tau)}{\left(1+y_{2 \varepsilon}(\cdot, t-\tau)\right)^{2}}\left|\nabla \kappa_{\varepsilon}\right|^{2} \nabla \kappa_{\varepsilon} \cdot \nabla y_{2 \varepsilon}(\cdot, t-\tau) \\
\leq & \frac{27}{32} M^{4} \int_{\Omega} \frac{1}{\left(1+y_{2 \varepsilon}(\cdot, t-\tau)\right)^{4}}\left|\nabla y_{1 \varepsilon}(\cdot, t-\tau)\right|^{4} \\
& +\frac{27}{32} M^{4} \int_{\Omega} \frac{y_{1 \varepsilon}^{4}(\cdot, t-\tau)}{\left(1+y_{2 \varepsilon}(\cdot, t-\tau)\right)^{8}}\left|\nabla y_{2 \varepsilon}(\cdot, t-\tau)\right|^{4} \\
\leq & \frac{27}{32} M^{4} \int_{\Omega}\left|\nabla y_{1 \varepsilon}(\cdot, t-\tau)\right|^{4}+\frac{27}{32} M^{4} K_{y}^{4} \int_{\Omega}\left|\nabla y_{2 \varepsilon}(\cdot, t-\tau)\right|^{4} \quad \text { for all } t>0
\end{aligned}
$$

because $y_{1 \varepsilon} \leq K_{y}$ by Lemma 3.6.

The above makes it necessary to characterize the evolution of $\int_{\Omega}\left|\nabla y_{1 \varepsilon}\right|^{4}$ and $\int_{\Omega}\left|\nabla y_{2 \varepsilon}\right|^{4}$ as well.

Lemma 4.8 There exists $C>0$ such that whenever $\varepsilon \in(0,1)$, the solution of (2.1) satisfies

$$
\begin{aligned}
\frac{d}{d t}\left\{\int_{\Omega}\left|\nabla y_{1 \varepsilon}\right|^{4}+\int_{\Omega}\left|\nabla y_{2 \varepsilon}\right|^{4}\right\} \leq & C \cdot\left\{1+\left\|\nabla l_{\varepsilon}(\cdot, t)\right\|_{L^{4}(\Omega)}+\left\|l_{\varepsilon}(\cdot, t)\right\|_{L^{\infty}(\Omega)}\right\} \times \\
& \times\left\{1+\int_{\Omega}\left|\nabla y_{1 \varepsilon}\right|^{4}+\int_{\Omega}\left|\nabla y_{2 \varepsilon}\right|^{4}\right\} \\
& +\int_{\Omega}\left|\nabla v_{\varepsilon}\right|^{4}
\end{aligned}
$$


for all $t>0$.

Proof. $\quad$ By (2.1), we have

$$
\nabla\left(\partial_{t} y_{1 \varepsilon}\right)=k_{1}\left(1-y_{1 \varepsilon}-y_{2 \varepsilon}\right) \nabla v_{\varepsilon}-k_{1} v_{\varepsilon} \nabla y_{1 \varepsilon}-k_{1} v_{\varepsilon} \nabla y_{2 \varepsilon}-k_{-1} \nabla y_{1 \varepsilon}
$$

and

$$
\nabla\left(\partial_{t} y_{2 \varepsilon}\right)=k_{2}\left(1-y_{1 \varepsilon}-y_{2 \varepsilon}\right) \nabla l_{\varepsilon}-k_{2} l_{\varepsilon} \nabla y_{1 \varepsilon}-k_{2} l_{\varepsilon} \nabla y_{2 \varepsilon}-k_{-2} \nabla y_{2 \varepsilon}
$$

in $\Omega \times(0, \infty)$, so that

$$
\begin{aligned}
\frac{1}{4} \frac{d}{d t} \int_{\Omega}\left|\nabla y_{1 \varepsilon}\right|^{4}+\frac{1}{4} \frac{d}{d t} \int_{\Omega}\left|\nabla y_{2 \varepsilon}\right|^{4}= & \int_{\Omega}\left|\nabla y_{1 \varepsilon}\right|^{2} \nabla y_{1 \varepsilon} \nabla\left(\partial_{t} y_{1 \varepsilon}\right)+\int_{\Omega}\left|\nabla y_{2 \varepsilon}\right|^{2} \nabla y_{2 \varepsilon} \nabla\left(\partial_{t} y_{2 \varepsilon}\right) \\
= & k_{1} \int_{\Omega}\left(1-y_{1 \varepsilon}-y_{2 \varepsilon}\right)\left|\nabla y_{1 \varepsilon}\right|^{2} \nabla y_{1 \varepsilon} \cdot \nabla v_{\varepsilon} \\
& -k_{1} \int_{\Omega} v_{\varepsilon}\left|\nabla y_{1 \varepsilon}\right|^{4}-k_{1} \int_{\Omega} v_{\varepsilon}\left|\nabla y_{1 \varepsilon}\right|^{2} \nabla y_{1 \varepsilon} \cdot \nabla y_{2 \varepsilon} \\
& -k_{-1} \int_{\Omega}\left|\nabla y_{1 \varepsilon}\right|^{4} \\
& +k_{2} \int_{\Omega}\left(1-y_{1 \varepsilon}-y_{2 \varepsilon}\right)\left|\nabla y_{2 \varepsilon}\right|^{2} \nabla y_{2 \varepsilon} \cdot \nabla l_{\varepsilon} \\
& -k_{2} \int_{\Omega} l_{\varepsilon}\left|\nabla y_{2 \varepsilon}\right|^{2} \nabla y_{1 \varepsilon} \cdot \nabla y_{2 \varepsilon}-k_{2} \int_{\Omega} l_{\varepsilon}\left|\nabla y_{2 \varepsilon}\right|^{4} \\
& -k_{-2} \int_{\Omega}\left|\nabla y_{2 \varepsilon}\right|^{4} \quad \text { for all } t>0 .
\end{aligned}
$$

Here we use Young's inequality and Lemma 3.6 to find $C_{1}>0$ such that

$$
\begin{aligned}
k_{1} \int_{\Omega}\left(1-y_{1 \varepsilon}-y_{2 \varepsilon}\right)\left|\nabla y_{1 \varepsilon}\right|^{2} \nabla y_{1 \varepsilon} \cdot \nabla v_{\varepsilon} & \leq k_{1}\left(1+K_{y}\right) \int_{\Omega}\left|\nabla y_{1 \varepsilon}\right|^{3}\left|\nabla v_{\varepsilon}\right| \\
& \leq \frac{1}{4} \int_{\Omega}\left|\nabla v_{\varepsilon}\right|^{4}+C_{1} \int_{\Omega}\left|\nabla y_{1 \varepsilon}\right|^{4}
\end{aligned}
$$

whereas by the same token along with Lemma 3.3 we obtain $C_{2}>0$ such that

$$
-k_{1} \int_{\Omega} v_{\varepsilon}\left|\nabla y_{1 \varepsilon}\right|^{2} \nabla y_{1 \varepsilon} \cdot \nabla y_{2 \varepsilon} \leq \int_{\Omega}\left|\nabla y_{1 \varepsilon}\right|^{4}+C_{2} \int_{\Omega}\left|\nabla y_{2 \varepsilon}\right|^{4} .
$$

As for the first of the integrals in (4.17) involving $l_{\varepsilon}$, we first apply the Hölder inequality and then Young's inequality to see that

$$
\begin{aligned}
k_{2} \int_{\Omega}\left(1-y_{1 \varepsilon}-y_{2 \varepsilon}\right)\left|\nabla y_{2 \varepsilon}\right|^{2} \nabla y_{2 \varepsilon} \cdot \nabla l_{\varepsilon} & \leq k_{2}\left(1+K_{y}\right)\left\|\nabla l_{\varepsilon}\right\|_{L^{4}(\Omega)}\left(\int_{\Omega}\left|\nabla y_{2 \varepsilon}\right|^{4}\right)^{\frac{3}{4}} \\
& \leq k_{2}\left(1+K_{y}\right)\left\|\nabla l_{\varepsilon}\right\|_{L^{4}(\Omega)} \cdot\left\{1+\int_{\Omega}\left|\nabla y_{2 \varepsilon}\right|^{4}\right\},
\end{aligned}
$$


and in the second we again use Young's inequality to estimate

$$
\begin{aligned}
-k_{2} \int_{\Omega} l_{\varepsilon}\left|\nabla y_{2 \varepsilon}\right|^{2} \nabla y_{1 \varepsilon} \cdot \nabla y_{2 \varepsilon}-k_{2} \int_{\Omega} l_{\varepsilon}\left|\nabla y_{2 \varepsilon}\right|^{4} & \leq k_{2} \int_{\Omega} l_{\varepsilon}\left|\nabla y_{1 \varepsilon}\right|^{4} \\
& \leq k_{2}\left\|l_{\varepsilon}\right\|_{L^{\infty}(\Omega)} \cdot \int_{\Omega}\left|\nabla y_{1 \varepsilon}\right|^{4} .
\end{aligned}
$$

In summary, (4.18)-(4.21) inserted into (4.17) readily prove (4.16).

We can now pass to the proof of the main result of this section.

Proof of Lemma 4.1. In light of Lemma 4.5, upon integration over $\Omega$ the inequality (4.11) from Lemma 4.4 becomes

$$
\begin{aligned}
\frac{d}{d t} \int_{\Omega} \frac{\kappa_{\varepsilon}\left|\nabla v_{\varepsilon}\right|^{2}}{1+v_{\varepsilon}}+ & 2 \varepsilon \int_{\Omega} \kappa_{\varepsilon}\left(1+v_{\varepsilon}\right)\left|D^{2} \ln \left(1+v_{\varepsilon}\right)\right|^{2} \\
\leq & -2 \lambda \int_{\Omega} \frac{\kappa_{\varepsilon} v_{\varepsilon}}{1+v_{\varepsilon}} \nabla c_{\varepsilon} \cdot \nabla v_{\varepsilon}-2 \lambda \int_{\Omega} \kappa_{\varepsilon} c_{\varepsilon} \frac{\left|\nabla v_{\varepsilon}\right|^{2}}{\left(1+v_{\varepsilon}\right)^{2}} \\
& +\left(2 \mu_{v}+1\right) K_{\kappa} \int_{\Omega} \frac{\left|\nabla v_{\varepsilon}\right|^{2}}{1+v_{\varepsilon}} \\
& -2 \varepsilon \int_{\Omega} \frac{1}{1+v_{\varepsilon}} \nabla \kappa_{\varepsilon} \cdot\left(D^{2} v_{\varepsilon} \cdot \nabla v_{\varepsilon}\right) \\
& +\varepsilon \int_{\Omega} \frac{1}{\left(1+v_{\varepsilon}\right)^{2}}\left|\nabla v_{\varepsilon}\right|^{2} \nabla \kappa_{\varepsilon} \cdot \nabla v_{\varepsilon} \quad \text { for all } t>0 .
\end{aligned}
$$

In order to estimate the two rightmost integrals appropriately, let us first apply Lemma 3.7 to find $C_{1}(T)>0$ such that

$$
\kappa_{\varepsilon}(x, t) \geq C_{1}(T) \quad \text { for all } x \in \Omega \text { and } t \in(0, T) .
$$

Then Lemma 4.6 ensures that

$$
\begin{aligned}
\int_{\Omega} \frac{\left|\nabla v_{\varepsilon}\right|^{4}}{\left(1+v_{\varepsilon}\right)^{3}} & \leq(2+\sqrt{n})^{2} \int_{\Omega}\left(1+v_{\varepsilon}\right)\left|D^{2} \ln \left(1+v_{\varepsilon}\right)\right|^{2} \\
& \leq C_{2}(T) \int_{\Omega} \kappa_{\varepsilon}\left(1+v_{\varepsilon}\right)\left|D^{2} \ln \left(1+v_{\varepsilon}\right)\right|^{2} \quad \text { for all } t \in(0, T)
\end{aligned}
$$

with $C_{2}(T):=\frac{(2+\sqrt{n})^{2}}{C_{1}(T)}$. Now using once again the pointwise identity

$$
\partial_{i j} v_{\varepsilon}=\left(1+v_{\varepsilon}\right) \partial_{i j} \ln \left(1+v_{\varepsilon}\right)+\frac{\partial_{i} v_{\varepsilon} \partial_{j} v_{\varepsilon}}{1+v_{\varepsilon}}, \quad(x, t) \in \Omega \times(0, \infty), i, j \in\{1, \ldots, n\},
$$

we obtain

$$
\begin{aligned}
-2 \varepsilon \int_{\Omega} \frac{1}{1+v_{\varepsilon}} \nabla \kappa_{\varepsilon} \cdot\left(D^{2} v_{\varepsilon} \cdot \nabla v_{\varepsilon}\right)+ & \varepsilon \int_{\Omega} \frac{1}{\left(1+v_{\varepsilon}\right)^{2}}\left|\nabla v_{\varepsilon}\right|^{2} \nabla \kappa_{\varepsilon} \cdot \nabla v_{\varepsilon} \\
= & -2 \varepsilon \int_{\Omega} \nabla \kappa_{\varepsilon} \cdot\left(D^{2} \ln \left(1+v_{\varepsilon}\right) \cdot \nabla v_{\varepsilon}\right) \\
& -\varepsilon \int_{\Omega} \frac{1}{\left(1+v_{\varepsilon}\right)^{2}}\left|\nabla v_{\varepsilon}\right|^{2} \nabla \kappa_{\varepsilon} \cdot \nabla v_{\varepsilon} \quad \text { for all } t>0
\end{aligned}
$$


where by Young's inequality, (4.23), (4.24) and Lemma 3.3 we find that

$$
\begin{aligned}
-2 \varepsilon \int_{\Omega} \nabla \kappa_{\varepsilon} \cdot\left(D^{2} \ln \left(1+v_{\varepsilon}\right) \cdot \nabla v_{\varepsilon}\right) \leq & \frac{\varepsilon}{2} \int_{\Omega} \kappa_{\varepsilon}\left(1+v_{\varepsilon}\right)\left|D^{2} \ln \left(1+v_{\varepsilon}\right)\right|^{2} \\
& +2 \varepsilon \int_{\Omega} \frac{1}{1+v_{\varepsilon}} \frac{\left|\nabla \kappa_{\varepsilon}\right|^{2}}{\kappa_{\varepsilon}}\left|\nabla v_{\varepsilon}\right|^{2} \\
\leq & \frac{\varepsilon}{2} \int_{\Omega} \kappa_{\varepsilon}\left(1+v_{\varepsilon}\right)\left|D^{2} \ln \left(1+v_{\varepsilon}\right)\right|^{2} \\
& +\frac{\varepsilon}{2 C_{2}(T)} \int_{\Omega} \frac{\left|\nabla v_{\varepsilon}\right|^{4}}{\left(1+v_{\varepsilon}\right)^{3}}+2 \varepsilon C_{2}(T) \int_{\Omega}\left(1+v_{\varepsilon}\right) \frac{\left|\nabla \kappa_{\varepsilon}\right|^{4}}{\kappa_{\varepsilon}^{2}} \\
\leq & \varepsilon \int_{\Omega} \kappa_{\varepsilon}\left(1+v_{\varepsilon}\right) \mid D^{2} \ln \left(1+\left.v_{\varepsilon}\right|^{2}+\frac{2 \varepsilon C_{2}(T)\left(1+K_{v}\right)}{C_{1}^{2}(T)} \int_{\Omega}\left|\nabla \kappa_{\varepsilon}\right|^{4}\right.
\end{aligned}
$$

for all $t \in(0, T)$. Similarly,

$$
\begin{aligned}
-\varepsilon \int_{\Omega} \frac{1}{\left(1+v_{\varepsilon}\right)^{2}}\left|\nabla v_{\varepsilon}\right|^{2} \nabla \kappa_{\varepsilon} \cdot \nabla v_{\varepsilon} & \leq \frac{\varepsilon}{2 C_{2}(T)} \int_{\Omega} \frac{\left|\nabla v_{\varepsilon}\right|^{4}}{\left(1+v_{\varepsilon}\right)^{3}}+\frac{27 \varepsilon C_{2}^{3}(T)}{32} \int_{\Omega}\left(1+v_{\varepsilon}\right)\left|\nabla \kappa_{\varepsilon}\right|^{4} \\
& \leq \frac{\varepsilon}{2} \int_{\Omega} \kappa_{\varepsilon}\left(1+v_{\varepsilon}\right)\left|D^{2} \ln \left(1+v_{\varepsilon}\right)\right|^{2}+\frac{27 \varepsilon C_{2}^{3}(T)\left(1+K_{v}\right)}{32} \int_{\Omega}\left|\nabla \kappa_{\varepsilon}\right|^{4}
\end{aligned}
$$

for all $t \in(0, T)$. Since clearly, again by $(4.23)$,

$$
\int_{\Omega} \frac{\left|\nabla v_{\varepsilon}\right|^{2}}{1+v_{\varepsilon}} \leq \frac{1}{C_{1}(T)} \int_{\Omega} \frac{\kappa_{\varepsilon}\left|\nabla v_{\varepsilon}\right|^{2}}{1+v_{\varepsilon}}
$$

from (4.22) we therefore obtain that

$$
\begin{aligned}
\frac{d}{d t} \int_{\Omega} \frac{\kappa_{\varepsilon}\left|\nabla v_{\varepsilon}\right|^{2}}{1+v_{\varepsilon}}+ & \frac{\varepsilon}{2} \int_{\Omega} \kappa_{\varepsilon}\left(1+v_{\varepsilon}\right)\left|D^{2} \ln \left(1+v_{\varepsilon}\right)\right|^{2} \\
\leq & -2 \lambda \int_{\Omega} \frac{\kappa_{\varepsilon} v_{\varepsilon}}{1+v_{\varepsilon}} \nabla c_{\varepsilon} \cdot \nabla v_{\varepsilon}-2 \lambda \int_{\Omega} \kappa_{\varepsilon} c_{\varepsilon} \frac{\left|\nabla v_{\varepsilon}\right|^{2}}{\left(1+v_{\varepsilon}\right)^{2}} \\
& +C_{4}(T) \int_{\Omega} \frac{\kappa_{\varepsilon}\left|\nabla v_{\varepsilon}\right|^{2}}{1+v_{\varepsilon}}+\varepsilon C_{4}(T) \int_{\Omega}\left|\nabla \kappa_{\varepsilon}\right|^{4} \quad \text { for all } t \in(0, T)
\end{aligned}
$$

with some $C_{4}(T)>0$. When multiplied by $\frac{1}{2 \lambda}$ and added to (4.4), this shows that there exists $C_{5}>0$ such that

$$
\begin{aligned}
\frac{d}{d t}\left\{\int_{\Omega} c_{\varepsilon} \ln c_{\varepsilon}+\frac{1}{2 \lambda} \int_{\Omega} \frac{\kappa_{\varepsilon}\left|\nabla v_{\varepsilon}\right|^{2}}{1+v_{\varepsilon}}\right\}+ & \frac{1}{2} \int_{\Omega} \frac{\kappa_{\varepsilon}}{1+c_{\varepsilon} v_{\varepsilon}} \frac{\left|\nabla c_{\varepsilon}\right|^{2}}{c_{\varepsilon}}+\int_{\Omega} \kappa_{\varepsilon} c_{\varepsilon} \frac{\left|\nabla v_{\varepsilon}\right|^{2}}{\left(1+v_{\varepsilon}\right)^{2}} \\
& +\frac{\mu_{c}}{2} \int_{\Omega} c_{\varepsilon}^{2} \ln \left(2+c_{\varepsilon}\right)+\varepsilon \int_{\Omega} \frac{\left|\nabla c_{\varepsilon}\right|^{2}}{c_{\varepsilon}}+\frac{\varepsilon}{2} \int_{\Omega} c_{\varepsilon}^{\theta} \ln \left(2+c_{\varepsilon}\right) \\
& +\frac{\varepsilon}{4 \lambda} \int_{\Omega} \kappa_{\varepsilon}\left(1+v_{\varepsilon}\right)\left|D^{2} \ln \left(1+v_{\varepsilon}\right)\right|^{2} \\
\leq & \frac{C_{4}(T)}{2 \lambda} \int_{\Omega} \frac{\kappa_{\varepsilon}\left|\nabla v_{\varepsilon}\right|^{2}}{1+v_{\varepsilon}}+\frac{1}{2} \int_{\Omega} \frac{c_{\varepsilon}\left(1+c_{\varepsilon} v_{\varepsilon}\right)}{\kappa_{\varepsilon}\left(1+c_{\varepsilon} l_{\varepsilon}\right)^{2}}\left|\nabla l_{\varepsilon}\right|^{2}+C_{5} \\
& +\frac{\varepsilon C_{4}(T)}{2 \lambda} \int_{\Omega}\left|\nabla \kappa_{\varepsilon}\right|^{4} \quad \text { for all } t \in(0, T) .
\end{aligned}
$$


Here the second integral on the right can be estimated using Lemma 3.5, which provides $C_{6}(T) \in$ $\left(0, K_{v}\right]$ fulfilling

$$
l_{\varepsilon}(x, t) \geq C_{6}(T) \quad \text { for all } x \in \Omega \text { and } t \in(0, T),
$$

so that by (4.23) and Lemma 3.3 we obtain the pointwise bound

$$
\begin{aligned}
\frac{c_{\varepsilon}\left(1+c_{\varepsilon} v_{\varepsilon}\right)}{\kappa_{\varepsilon}\left(1+c_{\varepsilon} l_{\varepsilon}\right)^{2}} & \leq \frac{c_{\varepsilon}\left(1+K_{v} c_{\varepsilon}\right)}{C_{1}(T)\left(1+C_{6}(T) c_{\varepsilon}\right)^{2}} \\
& =\frac{\frac{K_{v}}{C_{6}^{2}(T)} \cdot\left(C_{6}(T) c_{\varepsilon}\right) \cdot\left(\frac{C_{6}(T)}{K_{v}}+C_{6}(T) c_{\varepsilon}\right)}{C_{1}(T) \cdot\left(1+C_{6}(T) c_{\varepsilon}\right)^{2}} \\
& \leq \frac{K_{v}}{C_{1}(T) C_{6}^{2}(T)} \quad \text { for all } x \in \Omega \text { and } t \in(0, T) .
\end{aligned}
$$

By means of Lemma 3.5, we can thus find $C_{7}(T)>0$ such that

$$
\begin{aligned}
\frac{1}{2} \int_{\Omega} \frac{c_{\varepsilon}\left(1+c_{\varepsilon} v_{\varepsilon}\right)}{\kappa_{\varepsilon}\left(1+c_{\varepsilon} l_{\varepsilon}\right)^{2}}\left|\nabla l_{\varepsilon}\right|^{2} & \leq \frac{K_{v}}{2 C_{1}(T) C_{6}^{2}(T)} \int_{\Omega}\left|\nabla l_{\varepsilon}\right|^{2} \\
& \leq C_{7}(T) \quad \text { for all } t \in(0, T) .
\end{aligned}
$$

Now in order to compensate the last term on the right of (4.25), we recall that by Lemma 4.7 we have

$$
\frac{d}{d t} \int_{\Omega}\left|\nabla \kappa_{\varepsilon}\right|^{4} \leq C_{8} \cdot\left\{\int_{\Omega}\left|\nabla y_{1 \varepsilon}(\cdot, t-\tau)\right|^{4}+\int_{\Omega}\left|\nabla y_{2 \varepsilon}(\cdot, t-\tau)\right|^{4}\right\} \quad \text { for all } t>0
$$

and then invoke Lemma 4.8 to find $C_{9}>0$ fulfilling

$$
\begin{aligned}
\frac{d}{d t}\left\{\int_{\Omega}\left|\nabla y_{1 \varepsilon}\right|^{4}+\int_{\Omega}\left|\nabla y_{2 \varepsilon}\right|^{4}\right\} \leq \int_{\Omega}\left|\nabla v_{\varepsilon}\right|^{4}+C_{9} & \cdot\left\{1+\left\|\nabla l_{\varepsilon}(\cdot, t)\right\|_{L^{4}(\Omega)}+\left\|l_{\varepsilon}(\cdot, t)\right\|_{L^{\infty}(\Omega)}\right\} \times \\
& \times\left\{1+\int_{\Omega}\left|\nabla y_{1 \varepsilon}\right|^{4}+\int_{\Omega}\left|\nabla y_{2 \varepsilon}\right|^{4}\right\}
\end{aligned}
$$

for all $t>0$. Once more thanks to Lemma 3.3 and (4.24), we see that here

$$
\int_{\Omega}\left|\nabla v_{\varepsilon}\right|^{4} \leq\left(1+K_{v}\right)^{3} \int_{\Omega} \frac{\left|\nabla v_{\varepsilon}\right|^{4}}{\left(1+v_{\varepsilon}\right)^{3}} \leq\left(1+K_{v}\right)^{3} C_{2}(T) \int_{\Omega} \kappa_{\varepsilon}\left(1+v_{\varepsilon}\right)\left|D^{2} \ln \left(1+v_{\varepsilon}\right)\right|^{2},
$$

so that if we let $C_{10}(T):=\frac{1}{4 \lambda\left(1+K_{v}\right)^{3} C_{2}(T)}$ then from (4.25)-(4.29) we obtain

$$
\begin{aligned}
& \frac{d}{d t}\left\{\int_{\Omega} c_{\varepsilon} \ln c_{\varepsilon}+\frac{1}{2 \lambda} \int_{\Omega} \frac{\kappa_{\varepsilon}\left|\nabla v_{\varepsilon}\right|^{2}}{1+v_{\varepsilon}}+\varepsilon \int_{\Omega}\left|\nabla \kappa_{\varepsilon}\right|^{4}+\varepsilon C_{10}(T)\left(\int_{\Omega}\left|\nabla y_{1 \varepsilon}\right|^{4}+\int_{\Omega}\left|\nabla y_{2 \varepsilon}\right|^{4}\right)\right\} \\
& +\frac{1}{2} \int_{\Omega} \frac{\kappa_{\varepsilon}}{1+c_{\varepsilon} v_{\varepsilon}} \frac{\left|\nabla c_{\varepsilon}\right|^{2}}{c_{\varepsilon}}+\int_{\Omega} \kappa_{\varepsilon} c_{\varepsilon} \frac{\left|\nabla v_{\varepsilon}\right|^{2}}{\left(1+v_{\varepsilon}\right)^{2}}+\frac{\mu_{c}}{2} \int_{\Omega} c_{\varepsilon}^{2} \ln \left(2+c_{\varepsilon}\right)+\varepsilon \int_{\Omega} \frac{\left|\nabla c_{\varepsilon}\right|^{2}}{c_{\varepsilon}}+\frac{\varepsilon}{2} \int_{\Omega} c_{\varepsilon}^{\theta} \ln \left(2+c_{\varepsilon}\right) \\
& \leq \frac{C_{4}(T)}{2 \lambda} \int_{\Omega} \frac{\kappa_{\varepsilon}\left|\nabla v_{\varepsilon}\right|^{2}}{1+v_{\varepsilon}}+\varepsilon C_{8}\left\{\int_{\Omega}\left|\nabla y_{1 \varepsilon}(\cdot, t-\tau)\right|^{4}+\int_{\Omega}\left|\nabla y_{2 \varepsilon}(\cdot, t-\tau)\right|^{4}\right\} \\
& \quad+\varepsilon C_{9} C_{10}(T) \cdot\left\{1+\left\|\nabla l_{\varepsilon}(\cdot, t)\right\|_{L^{4}(\Omega)}+\left\|l_{\varepsilon}(\cdot, t)\right\|_{L^{\infty}(\Omega)}\right\} \cdot\left\{1+\int_{\Omega}\left|\nabla y_{1 \varepsilon}\right|^{4}+\int_{\Omega}\left|\nabla y_{2 \varepsilon}\right|^{4}\right\} \\
& +C_{7}(T)+C_{5}+\frac{\varepsilon C_{4}(T)}{2 \lambda} \int_{\Omega}\left|\nabla \kappa_{\varepsilon}\right|^{4} \quad \text { for all } t \in(0, T) .
\end{aligned}
$$


Now for $t \geq 0$ we introduce some nonnegative functions by letting

$$
\mathcal{E}_{\varepsilon}(t):=\int_{\Omega} c_{\varepsilon} \ln c_{\varepsilon}+\frac{1}{2 \lambda} \int_{\Omega} \frac{\kappa_{\varepsilon}\left|\nabla v_{\varepsilon}\right|^{2}}{1+v_{\varepsilon}}+\varepsilon \int_{\Omega}\left|\nabla \kappa_{\varepsilon}\right|^{4}+\varepsilon C_{10}(T) \cdot\left\{\int_{\Omega}\left|\nabla y_{1 \varepsilon}\right|^{4}+\int_{\Omega}\left|\nabla y_{2 \varepsilon}\right|^{4}\right\}+\frac{|\Omega|}{e}
$$

and

$$
f_{\varepsilon}(t):=1+\left\|\nabla l_{\varepsilon}(\cdot, t)\right\|_{L^{4}(\Omega)}+\left\|l_{\varepsilon}(\cdot, t)\right\|_{L^{\infty}(\Omega)}
$$

as well as

$$
\begin{aligned}
\mathcal{D}_{\varepsilon}(t):= & \frac{1}{2} \int_{\Omega} \frac{\kappa_{\varepsilon}}{1+c_{\varepsilon} v_{\varepsilon}} \frac{\left|\nabla c_{\varepsilon}\right|^{2}}{c_{\varepsilon}}+\int_{\Omega} \kappa_{\varepsilon} c_{\varepsilon} \frac{\left|\nabla v_{\varepsilon}\right|^{2}}{\left(1+v_{\varepsilon}\right)^{2}}+\frac{\mu_{c}}{2} \int_{\Omega} c_{\varepsilon}^{2} \ln \left(2+c_{\varepsilon}\right) \\
& +\varepsilon \int_{\Omega} \frac{\left|\nabla c_{\varepsilon}\right|^{2}}{c_{\varepsilon}}+\frac{\varepsilon}{2} \int_{\Omega} c_{\varepsilon}^{\theta} \ln \left(2+c_{\varepsilon}\right)
\end{aligned}
$$

and

$$
h_{\varepsilon}(t):=\int_{\Omega}\left|\nabla y_{1 \varepsilon}(\cdot, t)\right|^{4}+\int_{\Omega}\left|\nabla y_{2 \varepsilon}(\cdot, t)\right|^{4},
$$

and thereby see that with some adequately large $C_{11}(T)>0$, the inequality

$$
\frac{d}{d t} \mathcal{E}_{\varepsilon}(t)+\mathcal{D}_{\varepsilon}(t) \leq C_{11}(T) f_{\varepsilon}(t) \mathcal{E}_{\varepsilon}(t)+C_{11}(T) \varepsilon h_{\varepsilon}(t-\tau)
$$

holds for all $t \in(0, T)$. In particular, an ODE comparison shows that this implies

$$
\mathcal{E}_{\varepsilon}(t) \leq \mathcal{E}_{\varepsilon}(0) \cdot e^{C_{11}(T) \int_{0}^{t} f_{\varepsilon}(\sigma) d \sigma}+C_{11}(T) \int_{0}^{t} e^{C_{11}(T) \int_{s}^{t} f_{\varepsilon}(\sigma) d \sigma} \varepsilon h_{\varepsilon}(s-\tau) d s \quad \text { for all } t \in(0, T) .
$$

Here, $C_{12}:=\sup _{\varepsilon \in(0,1)} \mathcal{E}_{\varepsilon}(0)$ is finite by $(2.4)$, and Lemma 3.5 says that with some $C_{13}(T)>0$ we have

$$
\int_{0}^{T} f_{\varepsilon}(\sigma) d \sigma \leq C_{13}(T)
$$

for all $\varepsilon \in(0,1)$, where we have used that our assumption $n \leq 3$ ensures that $W^{2,2}(\Omega)$ is continuously embedded into both $W^{1,4}(\Omega)$ and $L^{\infty}(\Omega)$. Consequently, (4.32) yields

$$
\mathcal{E}_{\varepsilon}(t) \leq C_{12} e^{C_{11}(T) C_{13}(T)}+C_{11}(T) e^{C_{11}(T) C_{13}(T)} \cdot \int_{0}^{t} \varepsilon h_{\varepsilon}(s-\tau) d s \quad \text { for all } t \in(0, T) .
$$

By definition of $h_{\varepsilon}$ and $\mathcal{E}_{\varepsilon}$, however, we can estimate

$$
\begin{aligned}
\int_{0}^{t} \varepsilon h_{\varepsilon}(s-\tau) d s & \leq \int_{-\tau}^{0}\left\{\int_{\Omega}\left|\nabla y_{10 \varepsilon}\right|^{4}+\int_{\Omega}\left|\nabla y_{20 \varepsilon}\right|^{4}\right\}+\int_{0}^{(t-\tau)_{+}} \varepsilon h_{\varepsilon}(\sigma) d \sigma \\
& \leq C_{14}+\frac{1}{C_{10}(T)} \int_{0}^{t} \mathcal{E}_{\varepsilon}(\sigma) d \sigma \quad \text { for all } t \in(0, T)
\end{aligned}
$$


with $C_{14}:=\sup _{\varepsilon \in(0,1)}\left(\left\|\nabla y_{10 \varepsilon}\right\|_{L^{\infty}\left((-\tau, 0) ; L^{4}(\Omega)\right)}^{4}+\left\|\nabla y_{20 \varepsilon}\right\|_{L^{\infty}\left((-\tau, 0) ; L^{4}(\Omega)\right)}^{4}\right)$ being finite due to (2.4). Accordingly, (4.34) implies that with some $C_{15}(T)>0$ we have

$$
\mathcal{E}_{\varepsilon}(t) \leq C_{15}(T)+C_{15}(T) \int_{0}^{t} \mathcal{E}_{\varepsilon}(\sigma) d \sigma \quad \text { for all } t \in(0, T),
$$

whence the Grønwall lemma says that

$$
\mathcal{E}_{\varepsilon}(t) \leq C_{15}(T) \cdot e^{C_{15}(T) \cdot t} \leq C_{15}(T) \cdot e^{C_{15}(T) \cdot T} \quad \text { for all } t \in(0, T) .
$$

With this information at hand, we return to (4.31), use once more (4.33) and (4.35), and recall the definition of $\mathcal{D}_{\varepsilon}$ to readily end up with (4.1).

\section{Global weak solutions in the original problem}

Let us now specify our solution concept. In view of our intended compactness arguments, it will turn out to be convenient to formally rewrite $\nabla c=2 \sqrt{1+c} \cdot \nabla \sqrt{1+c}$.

Definition 5.1 Let $T>0$. Then by a weak solution of (1.1), (1.4), (1.5) we mean a collection of nonnegative functions

$$
\begin{aligned}
& c \in L^{2}(\Omega \times(0, T)) \quad \text { with } \quad \sqrt{1+c} \in L^{\frac{4}{3}}\left((0, T) ; W^{1, \frac{4}{3}}(\Omega)\right), \\
& v \in L^{\infty}(\Omega \times(0, T)) \cap L^{2}\left((0, T) ; W^{1,2}(\Omega)\right), \\
& l \in L^{2}\left((0, T) ; W^{1,2}(\Omega)\right), \\
& y_{1} \in L^{\infty}(\Omega \times(0, T)), \\
& y_{2} \in L^{\infty}(\Omega \times(0, T)) \quad \text { and } \\
& \kappa \in L^{\infty}(\Omega \times(0, T)),
\end{aligned}
$$

which satisfy for all $\varphi \in C_{0}^{\infty}(\bar{\Omega} \times[0, T))$

$$
\begin{aligned}
-\int_{0}^{T} \int_{\Omega} c \partial_{t} \varphi-\int_{\Omega} c_{0} \varphi(\cdot, 0)= & -2 \int_{0}^{T} \int_{\Omega} \frac{\kappa}{1+c v} \sqrt{1+c} \nabla \sqrt{1+c} \cdot \nabla \varphi+\int_{0}^{T} \int_{\Omega} \frac{\kappa v}{1+v} c \nabla v \cdot \nabla \varphi \\
& +\int_{0}^{T} \int_{\Omega} \frac{c}{1+c l} \nabla l \cdot \nabla \varphi+\mu_{c} \int_{0}^{T} \int_{\Omega} c\left(1-c-\eta_{1} v\right) \varphi
\end{aligned}
$$

and

$$
-\int_{0}^{T} \int_{\Omega} v \partial_{t} \varphi-\int_{\Omega} v_{0} \varphi(\cdot, 0)=\int_{0}^{T} \int_{\Omega}\left\{\mu_{v} v(1-v)-\lambda c v\right\} \cdot \varphi
$$

and

$$
-\int_{0}^{T} \int_{\Omega} l \partial_{t} \varphi-\int_{\Omega} l_{0} \varphi(\cdot, 0)=-\int_{0}^{T} \int_{\Omega} \nabla l \cdot \nabla \varphi+\int_{0}^{T} \int_{\Omega}(-l+c v) \varphi
$$


as well as

$$
-\int_{0}^{T} \int_{\Omega} y_{1} \partial_{t} \varphi-\int_{\Omega} y_{10} \varphi(\cdot, 0)=\int_{0}^{T} \int_{\Omega}\left\{k_{1}\left(1-y_{1}-y_{2}\right) v-k_{-1} y_{1}\right\} \varphi
$$

and

$$
-\int_{0}^{T} \int_{\Omega} y_{2} \partial_{t} \varphi-\int_{\Omega} y_{20} \varphi(\cdot, 0)=\int_{0}^{T} \int_{\Omega}\left\{k_{2}\left(1-y_{1}-y_{2}\right) l-k_{-2} y_{2}\right\} \varphi
$$

and

$$
-\int_{0}^{T} \int_{\Omega} \kappa \partial_{t} \varphi-\int_{\Omega} \kappa_{0} \varphi(\cdot, 0)=\int_{0}^{T} \int_{\Omega}\left\{-\kappa+\frac{M y_{1}(\cdot, t-\tau)}{1+y_{2}(\cdot, t-\tau)}\right\} \cdot \varphi
$$

$A$ global weak solution is a vector function $\left(c, v, l, y_{1}, y_{2}, \kappa\right): \Omega \times(0, \infty) \rightarrow \mathbb{R}^{6}$ which is a weak solution of (1.1) in $\Omega \times(0, T)$ for all $T>0$.

\subsection{Further $\varepsilon$-independent estimates for (2.1)}

Now besides the bounds provided in Section 3.2 and in Lemma 4.1, in our limit procedure we shall rely on some further estimates which basically derivate from the former. A particular goal will consist of establishing some strong compactness properties which allow to extract a.e. convergent subsequences.

\subsubsection{Estimates for $\sqrt{1+c_{\varepsilon}}$}

Let us first derive from Lemma 4.1 a bound for $\nabla \sqrt{1+c_{\varepsilon}}$ in a reflexive space.

Lemma 5.1 Let $T>0$. Then there exists $C(T)>0$ such that for any $\varepsilon \in(0,1)$ we have

$$
\int_{0}^{T}\left\|\sqrt{1+c_{\varepsilon}(\cdot, t)}\right\|_{W^{1, \frac{4}{3}(\Omega)}}^{\frac{4}{3}} d t \leq C(T)
$$

Proof. According to Lemma 4.1 and Lemma 3.7 we can find positive constants $C_{1}(T), C_{2}(T)$ and $C_{3}(T)$ such that

$$
\int_{0}^{T} \int_{\Omega} \frac{\kappa_{\varepsilon}}{1+c_{\varepsilon} v_{\varepsilon}} \frac{\left|\nabla c_{\varepsilon}\right|^{2}}{c_{\varepsilon}} \leq C_{1}(T)
$$

and

$$
\int_{0}^{T} \int_{\Omega} c_{\varepsilon}^{2} \leq C_{2}(T)
$$

as well as

$$
\kappa_{\varepsilon} \geq C_{3}(T) \quad \text { in } \Omega \times(0, T)
$$


for all $\varepsilon \in(0,1)$. Therefore using the Hölder inequality and Lemma 3.3 we can estimate

$$
\begin{aligned}
\int_{0}^{T} \int_{\Omega}\left|\nabla \sqrt{1+c_{\varepsilon}}\right|^{\frac{4}{3}} & =2^{-\frac{4}{3}} \int_{0}^{T} \int_{\Omega} \frac{\left|\nabla c_{\varepsilon}\right|^{\frac{4}{3}}}{\left(1+c_{\varepsilon}\right)^{\frac{2}{3}}} \\
& =2^{-\frac{4}{3}} \int_{0}^{T} \int_{\Omega}\left\{\frac{\kappa_{\varepsilon}}{1+c_{\varepsilon} v_{\varepsilon}} \frac{\left|\nabla c_{\varepsilon}\right|^{2}}{c_{\varepsilon}}\right\}^{\frac{2}{3}} \cdot\left\{\frac{1+c_{\varepsilon} v_{\varepsilon}}{\kappa_{\varepsilon}} \cdot \frac{c_{\varepsilon}}{1+c_{\varepsilon}}\right\}^{\frac{2}{3}} \\
& \leq 2^{-\frac{4}{3}} \cdot\left\{\int_{0}^{T} \int_{\Omega} \frac{\kappa_{\varepsilon}}{1+c_{\varepsilon} v_{\varepsilon}} \frac{\left|\nabla c_{\varepsilon}\right|^{2}}{c_{\varepsilon}}\right\}^{\frac{2}{3}} \cdot\left\{\int_{0}^{T} \int_{\Omega} \frac{\left(1+c_{\varepsilon} v_{\varepsilon}\right)^{2}}{\kappa_{\varepsilon}^{2}} \cdot \frac{c_{\varepsilon}^{2}}{\left(1+c_{\varepsilon}\right)^{2}}\right\}^{\frac{1}{3}} \\
& \leq 2^{-\frac{4}{3}} \cdot\left\{\int_{0}^{T} \int_{\Omega} \frac{\kappa_{\varepsilon}}{1+c_{\varepsilon} v_{\varepsilon}} \frac{\left|\nabla c_{\varepsilon}\right|^{2}}{c_{\varepsilon}}\right\}^{\frac{2}{3}} \cdot\left\{\frac{\left(1+K_{v}\right)^{2}}{C_{3}^{2}(T)} \int_{0}^{T} \int_{\Omega} c_{\varepsilon}^{2}\right\}^{\frac{1}{3}} \\
& \leq 2^{-\frac{4}{3}} \cdot C_{1}^{\frac{2}{3}}(T) \cdot\left\{\frac{\left(1+K_{v}\right)^{2}}{C_{3}^{2}(T)} \cdot C_{2}(T)\right\}^{\frac{1}{3}}
\end{aligned}
$$

which in view of Lemma 3.2 immediately proves (5.7).

In order to obtain a strong compactness property for $\sqrt{1+c_{\varepsilon}}$ from this, we need to find a suitable control for the respective time derivative. This will be possible only in a temporal $L^{1}$ framework.

Lemma 5.2 Let $k \in \mathbb{N}$ be such that $k>\frac{n+2}{2}$. Then for all $T>0$ there exists $C(T)>0$ such that

$$
\int_{0}^{T}\left\|\partial_{t} \sqrt{1+c_{\varepsilon}(\cdot, t)}\right\|_{\left(W_{0}^{k, 2}(\Omega)\right)^{\star}} d t \leq C(T)
$$

for all $\varepsilon \in(0,1)$.

Proof. $\quad$ Since $\partial_{t} \sqrt{1+c_{\varepsilon}}=\frac{\partial_{t} c_{\varepsilon}}{2 \sqrt{1+c_{\varepsilon}}}$ in $\Omega \times(0, \infty)$, for each fixed $t \in(0, T)$ and arbitrary $\psi \in C_{0}^{\infty}(\Omega)$, an integration by parts in (2.1) yields

$$
\begin{aligned}
2 \int_{\Omega} \partial_{t} \sqrt{1+c_{\varepsilon}} \cdot \psi= & \int_{\Omega}\left\{-\varepsilon \nabla c_{\varepsilon}-\frac{\kappa_{\varepsilon}}{1+c_{\varepsilon} v_{\varepsilon}} \nabla c_{\varepsilon}+\frac{\kappa_{\varepsilon} v_{\varepsilon}}{1+v_{\varepsilon}} c_{\varepsilon} \nabla v_{\varepsilon}+\frac{1}{1+c_{\varepsilon} l_{\varepsilon}} c_{\varepsilon} \nabla l_{\varepsilon}\right\} \cdot \nabla \frac{\psi}{\sqrt{1+c_{\varepsilon}}} \\
& +\mu_{c} \int_{\Omega} c_{\varepsilon}\left(1-c_{\varepsilon}-\eta_{1} v_{\varepsilon}\right) \cdot \frac{\psi}{\sqrt{1+c_{\varepsilon}}}-\varepsilon \int_{\Omega} c_{\varepsilon}^{\theta} \cdot \frac{\psi}{\sqrt{1+c_{\varepsilon}}} \\
= & \frac{\varepsilon}{2} \int_{\Omega} \frac{1}{{\sqrt{1+c_{\varepsilon}}}^{3}}\left|\nabla c_{\varepsilon}\right|^{2} \psi-\varepsilon \int_{\Omega} \frac{1}{\sqrt{1+c_{\varepsilon}}} \nabla c_{\varepsilon} \cdot \nabla \psi \\
& +\frac{1}{2} \int_{\Omega} \frac{\kappa_{\varepsilon}}{\left(1+c_{\varepsilon} v_{\varepsilon}\right){\sqrt{1+c_{\varepsilon}}}^{3}}\left|\nabla c_{\varepsilon}\right|^{2} \psi-\int_{\Omega} \frac{\kappa_{\varepsilon}}{\left(1+c_{\varepsilon} v_{\varepsilon}\right) \sqrt{1+c_{\varepsilon}}} \nabla c_{\varepsilon} \cdot \nabla \psi \\
& -\frac{1}{2} \int_{\Omega} \frac{\kappa_{\varepsilon} v_{\varepsilon} c_{\varepsilon}}{\left(1+v_{\varepsilon}\right){\sqrt{1+c_{\varepsilon}}}^{3}} \nabla c_{\varepsilon} \cdot \nabla v_{\varepsilon} \psi+\int_{\Omega} \frac{\kappa_{\varepsilon} v_{\varepsilon} c_{\varepsilon}}{\left(1+v_{\varepsilon}\right) \sqrt{1+c_{\varepsilon}}} \nabla v_{\varepsilon} \cdot \nabla \psi \\
& -\frac{1}{2} \int_{\Omega} \frac{c_{\varepsilon}}{\left(1+c_{\varepsilon} l_{\varepsilon}\right){\sqrt{1+c_{\varepsilon}}}^{3}} \nabla c_{\varepsilon} \cdot \nabla l_{\varepsilon} \psi+\int_{\Omega} \frac{c_{\varepsilon}}{\left(1+c_{\varepsilon} l_{\varepsilon}\right) \sqrt{1+c_{\varepsilon}}} \nabla l_{\varepsilon} \cdot \nabla \psi \\
& +\mu_{c} \int_{\Omega} c_{\varepsilon}\left(1-c_{\varepsilon}-\eta_{1} v_{\varepsilon}\right) \cdot \frac{\psi}{\sqrt{1+c_{\varepsilon}}}-\varepsilon \int_{\Omega} c_{\varepsilon}^{\theta} \cdot \frac{\psi}{\sqrt{1+c_{\varepsilon}}} .
\end{aligned}
$$


Here several straightforward estimations involving Young's inequality, Lemma 3.3 and Lemma 3.7 show that

$$
\left.\left|\frac{\varepsilon}{2} \int_{\Omega} \frac{1}{{\sqrt{1+c_{\varepsilon}}}^{3}}\right| \nabla c_{\varepsilon}\right|^{2} \psi \mid \leq\left(\frac{\varepsilon}{2} \int_{\Omega} \frac{\left|\nabla c_{\varepsilon}\right|^{2}}{c_{\varepsilon}}\right) \cdot\|\psi\|_{L^{\infty}(\Omega)}
$$

and

$$
\begin{aligned}
\left|-\varepsilon \int_{\Omega} \frac{1}{\sqrt{1+c_{\varepsilon}}} \nabla c_{\varepsilon} \cdot \nabla \psi\right| & \leq\left\{\varepsilon \int_{\Omega} \frac{\left|\nabla c_{\varepsilon}\right|^{2}}{c_{\varepsilon}}+\frac{\varepsilon}{4} \int_{\Omega} \frac{c_{\varepsilon}}{1+c_{\varepsilon}}\right\} \cdot\|\nabla \psi\|_{L^{\infty}(\Omega)} \\
& \leq\left\{\varepsilon \int_{\Omega} \frac{\left|\nabla c_{\varepsilon}\right|^{2}}{c_{\varepsilon}}+\frac{|\Omega|}{4}\right\} \cdot\|\nabla \psi\|_{L^{\infty}(\Omega)}
\end{aligned}
$$

and

$$
\left.\left|\frac{1}{2} \int_{\Omega} \frac{\kappa_{\varepsilon}}{\left(1+c_{\varepsilon} v_{\varepsilon}\right){\sqrt{1+c_{\varepsilon}}}^{3}}\right| \nabla c_{\varepsilon}\right|^{2} \psi \mid \leq\left(\frac{1}{2} \int_{\Omega} \frac{\kappa_{\varepsilon}}{1+c_{\varepsilon} v_{\varepsilon}} \frac{\left|\nabla c_{\varepsilon}\right|^{2}}{c_{\varepsilon}}\right) \cdot\|\psi\|_{L^{\infty}(\Omega)}
$$

as well as

$$
\begin{aligned}
\left|-\int_{\Omega} \frac{\kappa_{\varepsilon}}{\left(1+c_{\varepsilon} v_{\varepsilon}\right) \sqrt{1+c_{\varepsilon}}} \nabla c_{\varepsilon} \cdot \nabla \psi\right| & \leq\left\{\int_{\Omega} \frac{\kappa_{\varepsilon}}{1+c_{\varepsilon} v_{\varepsilon}} \frac{\left|\nabla c_{\varepsilon}\right|^{2}}{c_{\varepsilon}}+\frac{1}{4} \int_{\Omega} \frac{\kappa_{\varepsilon} c_{\varepsilon}}{\left(1+c_{\varepsilon} v_{\varepsilon}\right)\left(1+c_{\varepsilon}\right)}\right\} \cdot\|\nabla \psi\|_{L^{\infty}(\Omega)} \\
& \leq\left\{\int_{\Omega} \frac{\kappa_{\varepsilon}}{1+c_{\varepsilon} v_{\varepsilon}} \frac{\left|\nabla c_{\varepsilon}\right|^{2}}{c_{\varepsilon}}+\frac{K_{\kappa}|\Omega|}{4}\right\} \cdot\|\nabla \psi\|_{L^{\infty}(\Omega)}
\end{aligned}
$$

and

$$
\begin{aligned}
& \left|-\frac{1}{2} \int_{\Omega} \frac{\kappa_{\varepsilon} v_{\varepsilon} c_{\varepsilon}}{\left(1+v_{\varepsilon}\right) \sqrt{1+c_{\varepsilon}}} \nabla c_{\varepsilon} \cdot \nabla v_{\varepsilon} \psi\right| \\
& \leq\left\{\int_{\Omega} \frac{\kappa_{\varepsilon}}{1+c_{\varepsilon} v_{\varepsilon}} \frac{\left|\nabla c_{\varepsilon}\right|^{2}}{c_{\varepsilon}}+\frac{1}{16} \int_{\Omega} \frac{\kappa_{\varepsilon} v_{\varepsilon}^{2} c_{\varepsilon}^{3}\left(1+c_{\varepsilon} v_{\varepsilon}\right)}{\left(1+v_{\varepsilon}\right)^{2}\left(1+c_{\varepsilon}\right)^{3}}\left|\nabla v_{\varepsilon}\right|^{2}\right\} \cdot\|\psi\|_{L^{\infty}(\Omega)} \\
& \leq\left\{\int_{\Omega} \frac{\kappa_{\varepsilon}}{1+c_{\varepsilon} v_{\varepsilon}} \frac{\left|\nabla c_{\varepsilon}\right|^{2}}{c_{\varepsilon}}+\frac{K_{v}^{2}\left(1+K_{v}\right)}{16} \int_{\Omega} \kappa_{\varepsilon} c_{\varepsilon} \frac{\left|\nabla v_{\varepsilon}\right|^{2}}{\left(1+v_{\varepsilon}\right)^{2}}\right\} \cdot\|\psi\|_{L^{\infty}(\Omega)}
\end{aligned}
$$

and

$$
\begin{aligned}
\left|\int_{\Omega} \frac{\kappa_{\varepsilon} v_{\varepsilon} c_{\varepsilon}}{\left(1+v_{\varepsilon}\right) \sqrt{1+c_{\varepsilon}}} \nabla v_{\varepsilon} \cdot \nabla \psi\right| & \leq\left\{\int_{\Omega} \kappa_{\varepsilon} c_{\varepsilon} \frac{\left|\nabla v_{\varepsilon}\right|^{2}}{\left(1+v_{\varepsilon}\right)^{2}}+\frac{1}{4} \int_{\Omega} \frac{\kappa_{\varepsilon} v_{\varepsilon}^{2} c_{\varepsilon}}{1+c_{\varepsilon}}\right\} \cdot\|\nabla \psi\|_{L^{\infty}(\Omega)} \\
& \leq\left\{\int_{\Omega} \kappa_{\varepsilon} c_{\varepsilon} \frac{\left|\nabla v_{\varepsilon}\right|^{2}}{\left(1+v_{\varepsilon}\right)^{2}}+\frac{K_{\kappa} K_{v}^{2}|\Omega|}{4}\right\} \cdot\|\nabla \psi\|_{L^{\infty}(\Omega)} .
\end{aligned}
$$

Now with $C_{1}(T) \in(0,1)$ and $C_{2}(T) \in(0,1)$, as provided by Lemma 3.7 and Lemma 3.5, such that $\kappa_{\varepsilon} \geq C_{1}(T)$ and $l_{\varepsilon} \geq C_{2}(T)$ in $\Omega \times(0, T)$, we similarly obtain

$$
\begin{aligned}
\mid-\frac{1}{2} \int_{\Omega} & \frac{c_{\varepsilon}}{\left(1+c_{\varepsilon} l_{\varepsilon}\right) \sqrt{1+c_{\varepsilon}}} \nabla c_{\varepsilon} \cdot \nabla l_{\varepsilon} \psi \mid \\
& \leq\left\{\int_{\Omega}\left|\nabla l_{\varepsilon}\right|^{2}+\frac{1}{16} \int_{\Omega} \frac{c_{\varepsilon}^{2}}{\left(1+c_{\varepsilon} l_{\varepsilon}\right)^{2}\left(1+c_{\varepsilon}\right)^{3}}\left|\nabla c_{\varepsilon}\right|^{2}\right\} \cdot\|\psi\|_{L^{\infty}(\Omega)} \\
& \leq\left\{\int_{\Omega}\left|\nabla l_{\varepsilon}\right|^{2}+\frac{1+K_{v}}{16 C_{1}(T) C_{2}(T)} \int_{\Omega} \frac{\kappa_{\varepsilon}}{1+c_{\varepsilon} v_{\varepsilon}} \frac{\left|\nabla c_{\varepsilon}\right|^{2}}{c_{\varepsilon}}\right\} \cdot\|\psi\|_{L^{\infty}(\Omega)}
\end{aligned}
$$


and

$$
\begin{aligned}
\left|\int_{\Omega} \frac{c_{\varepsilon}}{\left(1+c_{\varepsilon} l_{\varepsilon}\right) \sqrt{1+c_{\varepsilon}}} \nabla l_{\varepsilon} \cdot \nabla \psi\right| & \leq\left\{\int_{\Omega}\left|\nabla l_{\varepsilon}\right|^{2}+\frac{1}{4} \int_{\Omega} \frac{c_{\varepsilon}^{2}}{\left(1+c_{\varepsilon} l_{\varepsilon}\right)^{2}\left(1+c_{\varepsilon}\right)}\right\} \cdot\|\nabla \psi\|_{L^{\infty}(\Omega)} \\
& \leq\left\{\int_{\Omega}\left|\nabla l_{\varepsilon}\right|^{2}+\frac{|\Omega|}{4 C_{2}(T)}\right\} \cdot\|\nabla \psi\|_{L^{\infty}(\Omega)} .
\end{aligned}
$$

Since clearly

$$
\left|\mu_{c} \int_{\Omega} c_{\varepsilon}\left(1-c_{\varepsilon}-\eta_{1} v_{\varepsilon}\right) \cdot \frac{\psi}{\sqrt{1+c_{\varepsilon}}}\right| \leq \mu_{c}\left\{m+\int_{\Omega} c_{\varepsilon}^{2}+\eta_{1} K_{v} m\right\} \cdot\|\psi\|_{L^{\infty}(\Omega)}
$$

by Lemma 3.2 and

$$
\left|-\varepsilon \int_{\Omega} c_{\varepsilon}^{\theta} \cdot \frac{\psi}{\sqrt{1+c_{\varepsilon}}}\right| \leq\left(\varepsilon \int_{\Omega} c_{\varepsilon}^{\theta}\right) \cdot\|\psi\|_{L^{\infty}(\Omega)},
$$

from (5.9) we obtain altogether

$$
\left|\int_{\Omega} \partial_{t} \sqrt{1+c_{\varepsilon}(\cdot, t)} \cdot \psi\right| \leq f_{\varepsilon}(t) \cdot\|\psi\|_{W^{1, \infty}(\Omega)} \quad \text { for all } \psi \in C_{0}^{\infty}(\Omega),
$$

where

$$
\begin{aligned}
f_{\varepsilon}(t):=C_{3}(T) \cdot & \left\{\varepsilon \int_{\Omega} \frac{\left|\nabla c_{\varepsilon}\right|^{2}}{c_{\varepsilon}}+\int_{\Omega} \frac{\kappa_{\varepsilon}}{1+c_{\varepsilon} v_{\varepsilon}} \frac{\left|\nabla c_{\varepsilon}\right|^{2}}{c_{\varepsilon}}+\int_{\Omega} \kappa_{\varepsilon} c_{\varepsilon} \frac{\left|\nabla v_{\varepsilon}\right|^{2}}{\left(1+v_{\varepsilon}\right)^{2}}+\int_{\Omega}\left|\nabla l_{\varepsilon}\right|^{2}\right. \\
& \left.+\int_{\Omega} c_{\varepsilon}^{2}+\varepsilon \int_{\Omega} c_{\varepsilon}^{\theta}+1\right\}
\end{aligned}
$$

for $t \in(0, T)$ and $\varepsilon \in(0,1)$, with some suitably large $C_{3}(T)>0$.

Now since $k>\frac{n+2}{2}$ warrants that $W_{0}^{k, 2}(\Omega)$ is continuously embedded into $W^{1, \infty}(\Omega)$, (5.10) ensures that with some $C_{4}>0$ we have

$$
\left\|\partial_{t} \sqrt{1+c_{\varepsilon}(\cdot, t)}\right\|_{\left(W_{0}^{k, 2}(\Omega)\right)^{\star}}=\sup _{\substack{\psi \in C_{0}^{\infty}(\Omega) \\\|\psi\|_{W_{0}^{k, 2}(\Omega)} \leq 1}}\left|\int_{\Omega} \partial_{t} \sqrt{1+c_{\varepsilon}(\cdot, t)} \cdot \psi\right| \leq C_{4} f_{\varepsilon}(t)
$$

for all $t \in(0, T)$ and each $\varepsilon \in(0,1)$. A combination of Lemma 3.2, Lemma 3.5, and Lemma 4.1 yields the existence of a $C_{5}(T)>0$ fulfilling

$$
\int_{0}^{T} f_{\varepsilon}(t) d t \leq C_{5}(T) \quad \text { for all } \varepsilon \in(0,1)
$$

which proves (5.8).

Now the latter two lemmata lead in a standard way to the following.

Lemma 5.3 For each $T>0$,

$$
\left(\sqrt{1+c_{\varepsilon}}\right)_{\varepsilon \in(0,1)} \text { is strongly precompact in } L^{\frac{4}{3}}(\Omega \times(0, T)) \text {. }
$$

Proof. This is a direct consequence of Lemma 5.1, Lemma 5.2, and a variant of the Aubin-Lions lemma ([37, Theorem 2.3 and Remark 2.1 in Chapter III]), where we use the compactness of the embedding $W^{1, \frac{4}{3}}(\Omega) \hookrightarrow L^{\frac{4}{3}}(\Omega)$ and the fact that $\left(W_{0}^{k, 2}(\Omega)\right)^{\star}$ is a Hilbert space for any $k \in \mathbb{N}$. 


\subsubsection{Estimates for $v_{\varepsilon}, y_{1 \varepsilon}, y_{2 \varepsilon}$ and $\kappa_{\varepsilon}$}

For the control of the gradient of all the components related to ODEs in (1.1), Lemma 4.1 will be essential; indeed, as a first consequence we note the following.

Lemma 5.4 Let $T>0$. Then there exists $C(T)>0$ such that

$$
\sup _{t \in(0, T)} \int_{\Omega}\left|\nabla v_{\varepsilon}(\cdot, t)\right|^{2} \leq C(T)
$$

for all $\varepsilon \in(0,1)$.

Proof. $\quad$ From Lemma 4.1 we obtain $C_{1}(T)>0$ such that

$$
\sup _{t \in(0, T)} \int_{\Omega} \frac{\kappa_{\varepsilon}\left|\nabla v_{\varepsilon}\right|^{2}}{1+v_{\varepsilon}} \leq C_{1}(T),
$$

whereas Lemma 3.7 provides $C_{2}(T)>0$ fulfilling

$$
\kappa_{\varepsilon} \geq C_{2}(T) \quad \text { in } \Omega \times(0, T) .
$$

Therefore, by Lemma 3.3 we conclude that

$$
\int_{\Omega}\left|\nabla v_{\varepsilon}\right|^{2} \leq \frac{1+K_{v}}{C_{2}(T)} \int_{\Omega} \frac{\kappa_{\varepsilon}\left|\nabla v_{\varepsilon}\right|^{2}}{1+v_{\varepsilon}} \leq \frac{\left(1+K_{v}\right) C_{1}(T)}{C_{2}(T)}
$$

for all $t \in(0, T)$ and any $\varepsilon \in(0,1)$.

This result entails corresponding bounds for $y_{1 \varepsilon}$ and $y_{2 \varepsilon}$ :

Lemma 5.5 For all $T>0$ there exists $C(T)>0$ such that

$$
\sup _{t \in(0, T)}\left\{\int_{\Omega}\left|\nabla y_{1 \varepsilon}(\cdot, t)\right|^{2}+\int_{\Omega}\left|\nabla y_{2 \varepsilon}(\cdot, t)\right|^{2}\right\} \leq C(T)
$$

for all $\varepsilon \in(0,1)$.

Proof. We first proceed in much the same manner as in Lemma 4.8 to derive the identities

$$
\begin{aligned}
\frac{1}{2} \frac{d}{d t} \int_{\Omega}\left|\nabla y_{1 \varepsilon}\right|^{2}= & \int_{\Omega} \nabla y_{1 \varepsilon} \cdot \nabla\left(\partial_{t} y_{1 \varepsilon}\right) \\
= & k_{1} \int_{\Omega}\left(1-y_{1 \varepsilon}-y_{2 \varepsilon}\right) \nabla y_{1 \varepsilon} \cdot \nabla v_{\varepsilon}-k_{1} \int_{\Omega} v_{\varepsilon}\left|\nabla y_{1 \varepsilon}\right|^{2}-k_{1} \int_{\Omega} v_{\varepsilon} \nabla y_{1 \varepsilon} \cdot \nabla y_{2 \varepsilon} \\
& -k_{-1} \int_{\Omega}\left|\nabla y_{1 \varepsilon}\right|^{2}
\end{aligned}
$$

and

$$
\begin{aligned}
\frac{1}{2} \frac{d}{d t} \int_{\Omega}\left|\nabla y_{2 \varepsilon}\right|^{2}= & k_{2} \int_{\Omega}\left(1-y_{1 \varepsilon}-y_{2 \varepsilon}\right) \nabla y_{2 \varepsilon} \cdot \nabla l_{\varepsilon}-k_{2} \int_{\Omega} l_{\varepsilon} \nabla y_{1 \varepsilon} \cdot \nabla y_{2 \varepsilon} \\
& -k_{2} \int_{\Omega} l_{\varepsilon}\left|\nabla y_{2 \varepsilon}\right|^{2}-k_{-2} \int_{\Omega}\left|\nabla y_{2 \varepsilon}\right|^{2}
\end{aligned}
$$


for all $t>0$, where using Young's inequality, Lemma 3.6 and Lemma 3.3 we can estimate

$$
k_{1} \int_{\Omega}\left(1-y_{1 \varepsilon}-y_{2 \varepsilon}\right) \nabla y_{1 \varepsilon} \cdot \nabla v_{\varepsilon} \leq \int_{\Omega}\left|\nabla y_{1 \varepsilon}\right|^{2}+\frac{k_{1}^{2}\left(1+K_{y}^{2}\right)}{4} \int_{\Omega}\left|\nabla v_{\varepsilon}\right|^{2}
$$

and

$$
-k_{1} \int_{\Omega} v_{\varepsilon} \nabla y_{1 \varepsilon} \cdot \nabla y_{2 \varepsilon} \leq \int_{\Omega}\left|\nabla y_{1 \varepsilon}\right|^{2}+\frac{k_{1}^{2} K_{v}^{2}}{4} \int_{\Omega}\left|\nabla y_{2 \varepsilon}\right|^{2}
$$

as well as

$$
k_{2} \int_{\Omega}\left(1-y_{1 \varepsilon}-y_{2 \varepsilon}\right) \nabla y_{2 \varepsilon} \cdot \nabla l_{\varepsilon} \leq \int_{\Omega}\left|\nabla y_{2 \varepsilon}\right|^{2}+\frac{k_{2}^{2}\left(1+K_{y}^{2}\right)}{4} \int_{\Omega}\left|\nabla l_{\varepsilon}\right|^{2} .
$$

Again by Young's inequality,

$$
-k_{2} \int_{\Omega} l_{\varepsilon} \nabla y_{1 \varepsilon} \cdot \nabla y_{2 \varepsilon}-k_{2} \int_{\Omega} l_{\varepsilon}\left|\nabla y_{2 \varepsilon}\right|^{2} \leq \frac{k_{2}}{4}\left\|l_{\varepsilon}(\cdot, t)\right\|_{L^{\infty}(\Omega)} \cdot \int_{\Omega}\left|\nabla y_{1 \varepsilon}\right|^{2},
$$

whence adding (5.13) to (5.14) we see that with some $C_{1}>0$ we have

$$
\begin{aligned}
\frac{d}{d t}\left\{\int_{\Omega}\left|\nabla y_{1 \varepsilon}\right|^{2}+\int_{\Omega}\left|\nabla y_{2 \varepsilon}\right|^{2}\right\} \leq & C_{1} \cdot\left\{1+\left\|l_{\varepsilon}(\cdot, t)\right\|_{L^{\infty}(\Omega)}\right\} \cdot\left\{\int_{\Omega}\left|\nabla y_{1 \varepsilon}\right|^{2}+\int_{\Omega}\left|\nabla y_{2 \varepsilon}\right|^{2}\right\} \\
& +C_{1} \int_{\Omega}\left|\nabla v_{\varepsilon}\right|^{2}+C_{1} \int_{\Omega}\left|\nabla l_{\varepsilon}\right|^{2} \quad \text { for all } t>0 .
\end{aligned}
$$

Now due to Lemmata 5.4 and 3.5 we can find $C_{2}(T)>0$ such that $\int_{0}^{T} \int_{\Omega}\left(\left|\nabla v_{\varepsilon}\right|^{2}+\left|\nabla l_{\varepsilon}\right|^{2}\right) \leq C_{2}(T)$. Moreover, thanks to the Cauchy-Schwarz inequality, Lemma 3.5, and the fact that $W^{2,2}(\Omega) \hookrightarrow L^{\infty}(\Omega)$ by our assumption $n \leq 3$,

$$
\int_{0}^{T}\left\|l_{\varepsilon}(\cdot, t)\right\|_{L^{\infty}(\Omega)} d t \leq C_{3} T^{\frac{1}{2}}\left(\int_{0}^{T}\left\|l_{\varepsilon}(\cdot, t)\right\|_{W^{2,2}(\Omega)}^{2} d t\right)^{\frac{1}{2}} \leq C_{4}(T)
$$

for all $\varepsilon \in(0,1)$ with some $C_{3}>0$ and $C_{4}(T)>0$. Hence, integrating (5.15) easily yields (5.12).

This result implies in turn an estimate for $\int_{\Omega}\left|\nabla \kappa_{\varepsilon}\right|^{2}$ :

Lemma 5.6 Given $T>0$, we can find $C(T)>0$ such that

$$
\sup _{t \in(0, T)} \int_{\Omega}\left|\nabla \kappa_{\varepsilon}(\cdot, t)\right|^{2} \leq C(T)
$$

for all $\varepsilon \in(0,1)$.

Proof. Writing $z_{\varepsilon}(x, t):=\frac{M y_{1 \varepsilon}(x, t-\tau)}{1+y_{2 \varepsilon}(x, t-\tau)}$ for $(x, t) \in \Omega \times(0, \infty)$ and $\varepsilon \in(0,1)$, from Lemma 5.5 and Lemma 3.6 we easily infer the existence of $C_{1}(T)>0$ such that

$$
\int_{\Omega}\left|\nabla z_{\varepsilon}(\cdot, t)\right|^{2} \leq C_{1}(T) \quad \text { for all } t \in(0, T) .
$$


Thus by (2.1) and Young's inequality,

$$
\frac{1}{2} \frac{d}{d t} \int_{\Omega}\left|\nabla \kappa_{\varepsilon}\right|^{2}=-\int_{\Omega}\left|\nabla \kappa_{\varepsilon}\right|^{2}+\int_{\Omega} \nabla \kappa_{\varepsilon} \cdot \nabla z_{\varepsilon} \leq \frac{1}{4} \int_{\Omega}\left|\nabla z_{\varepsilon}\right|^{2} \leq \frac{C_{1}(T)}{4} \quad \text { for all } t \in(0, T),
$$

and (5.16) is immediate.

Now some bounds for the time derivatives of the approximations to the non-diffusible components in (1.1) are summarized in the following.

Lemma 5.7 There exists $C>0$ such that for all $\varepsilon \in(0,1)$ we have

$$
\begin{aligned}
& \int_{0}^{T}\left\|\partial_{t} v_{\varepsilon}(\cdot, t)\right\|_{L^{2}(\Omega)}^{2} d t \leq C(1+T) \quad \text { for all } T>0, \\
& \sup _{t>0}\left\|\partial_{t} y_{1 \varepsilon}(\cdot, t)\right\|_{L^{\infty}(\Omega)} \leq C, \\
& \int_{0}^{T}\left\|\partial_{t} y_{2 \varepsilon}(\cdot, t)\right\|_{L^{\infty}(\Omega)}^{2} d t \leq C(1+T) \quad \text { for all } T>0 \quad \text { and } \\
& \sup _{t>0}\left\|\partial_{t} \kappa_{\varepsilon}(\cdot, t)\right\|_{L^{\infty}(\Omega)} \leq C .
\end{aligned}
$$

Proof. The statements (5.18) and (5.20) are obvious from Lemma 3.3, Lemma 3.6, and Lemma 3.7. Likewise, (5.19) results upon recalling Lemma 3.5, because for some $C_{1}>0$ we have

$$
\int_{0}^{T}\left\|l_{\varepsilon}(\cdot, t)\right\|_{L^{\infty}(\Omega)}^{2} d t \leq C_{1} \int_{0}^{T}\left\|l_{\varepsilon}(\cdot, t)\right\|_{W^{2,2}(\Omega)}^{2} d t \quad \text { for all } T>0
$$

by the Sobolev embedding theorem and, again, the fact that $n \leq 3$. Finally, to derive (5.17) we multiply the second equation in (2.1) by $\partial_{t} v_{\varepsilon}$ to obtain

$$
\int_{\Omega}\left(\partial_{t} v_{\varepsilon}\right)^{2}+\frac{d}{d t}\left\{\frac{\varepsilon}{2} \int_{\Omega}\left|\nabla v_{\varepsilon}\right|^{2}-\frac{\mu_{v}}{2} \int_{\Omega} v_{\varepsilon}^{2}+\frac{\mu_{v}}{3} \int_{\Omega} v_{\varepsilon}^{3}\right\}=-\lambda \int_{\Omega} c_{\varepsilon} v_{\varepsilon} \partial_{t} v_{\varepsilon} \quad \text { for all } t>0 .
$$

Since by Young's inequality and Lemma 3.3,

$$
-\lambda \int_{\Omega} c_{\varepsilon} v_{\varepsilon} \partial_{t} v_{\varepsilon} \leq \frac{1}{2} \int_{\Omega}\left(\partial_{t} v_{\varepsilon}\right)^{2}+\frac{\lambda^{2} K_{v}^{2}}{2} \int_{\Omega} c_{\varepsilon}^{2} \quad \text { for all } t>0,
$$

using Lemma 3.2 we readily arrive at (5.17).

Combining the above lemmata, we directly obtain strong precompactness of all the considered solution components in $L_{l o c}^{2}(\bar{\Omega} \times[0, \infty))$.

Corollary 5.8 Let $T>0$. Then

$\left(l_{\varepsilon}\right)_{\varepsilon \in(0,1)},\left(v_{\varepsilon}\right)_{\varepsilon \in(0,1)},\left(y_{1 \varepsilon}\right)_{\varepsilon \in(0,1)},\left(y_{2 \varepsilon}\right)_{\varepsilon \in(0,1)}$ and $\left(\kappa_{\varepsilon}\right)_{\varepsilon \in(0,1)}$ are strongly precompact in $L^{2}(\Omega \times(0, T))$.

Proof. This is an evident by-product of Lemma 3.5, Lemma 5.4, Lemma 5.5, and Lemma 5.6 when combined with Lemma 5.7 and the Aubin-Lions lemma ([37, Theorem 2.1 in Chapter III]). 


\subsubsection{Further results on strong precompactness}

Now thanks to the presence of the logarithmic factor in the second last integral on the left of (4.1) we can apply the Dunford-Pettis theorem to derive the following further strong compactness results which will be essential in our passage to the limit $\varepsilon \searrow 0$.

Lemma 5.9 Let $T>0$. Then

$$
\left(c_{\varepsilon}\right)_{\varepsilon \in(0,1)} \text { is relatively compact with respect to the strong topology in } L^{2}(\Omega \times(0, T)) \text {, }
$$

and moreover

$$
\left(\frac{\kappa_{\varepsilon} \sqrt{1+c_{\varepsilon}}}{1+c_{\varepsilon} v_{\varepsilon}}\right)_{\varepsilon \in(0,1)} \quad \text { is relatively compact with respect to the strong topology in } L^{4}(\Omega \times(0, T)) \text {. }
$$

Proof. First, Lemma 4.1 provides $C_{1}(T)>0$ such that

$$
\int_{0}^{T} \int_{\Omega} c_{\varepsilon}^{2} \ln \left(2+c_{\varepsilon}\right) \leq C_{1}(T) \quad \text { for all } \varepsilon \in(0,1)
$$

Therefore, the Dunford-Pettis theorem $([1, \mathrm{~A} 6.14])$ asserts that $\left(c_{\varepsilon}^{2}\right)_{\varepsilon \in(0,1)}$ is relatively compact in $L^{1}(\Omega \times(0, T))$ with respect to the weak topology therein. This means that given $\left(\varepsilon_{j}\right)_{j \in \mathbb{N}} \subset(0,1)$ we can find a subsequence $\left(\varepsilon_{j i}\right)_{i \in \mathbb{N}}$ such that $c_{\varepsilon_{j_{i}}}^{2} \rightarrow z_{1}$ in $L^{1}(\Omega \times(0, T))$ as $i \rightarrow \infty$ with some $z_{1} \in L^{1}(\Omega \times(0, T))$. Upon a further extraction if necessary, by (5.23) and Lemma 5.3 we may assume that also $c_{\varepsilon_{j_{i}}} \rightarrow z_{2}$ in $L^{2}(\Omega \times(0, T))$ and $c_{\varepsilon_{j_{i}}} \rightarrow c$ a.e. in $\Omega \times(0, T)$ as $i \rightarrow \infty$ with a certain $z_{2} \in L^{2}(\Omega \times(0, T))$ and some measurable $c: \Omega \times(0, T) \rightarrow \mathbb{R}$, whence an application of Egorov's theorem ensures that $z_{2}=c$ and $z_{1}=c^{2}$. As thus $c_{\varepsilon_{j_{i}}}^{2} \rightarrow c^{2}$ in $L^{1}(\Omega \times(0, T))$ as $i \rightarrow \infty$, using constant test functions here we infer that $\int_{0}^{T} \int_{\Omega} c_{\varepsilon_{j_{i}}}^{2} \rightarrow \int_{0}^{T} \int_{\Omega} c^{2}$ and hence conclude from $c_{\varepsilon_{j_{i}}} \rightarrow c$ in $L^{2}(\Omega \times(0, T))$ that in fact $c_{\varepsilon} \rightarrow c$ in $L^{2}(\Omega \times(0, T))$ as $i \rightarrow \infty$, which proves (5.21).

To verify (5.22), we proceed similarly, noting first that (5.23) and Lemma 3.7 imply that $w_{\varepsilon}:=\frac{\kappa_{\varepsilon} \sqrt{1+c_{\varepsilon}}}{1+c_{\varepsilon} v_{\varepsilon}}$ satisfies

$$
\int_{0}^{T} \int_{\Omega} w_{\varepsilon}^{4} \ln \left(2+w_{\varepsilon}^{2}\right) \leq K_{\kappa}^{4} \int_{0}^{T} \int_{\Omega}\left(1+c_{\varepsilon}\right)^{2} \ln \left(2+K_{\kappa}^{2}\left(1+c_{\varepsilon}\right)\right) \leq C_{2}(T) \quad \text { for all } \varepsilon \in(0,1)
$$

with a certain $C_{2}(T)>0$. This entails that with respect to the corresponding weak topologies, $\left(w_{\varepsilon}\right)_{\varepsilon \in(0,1)}$ is relatively compact in $L^{4}(\Omega \times(0, T))$ and $\left(w_{\varepsilon}^{4}\right)_{\varepsilon \in(0,1)}$ is relatively compact in $L^{1}(\Omega \times(0, T))$, the latter again thanks to the Dunford-Pettis theorem. Now since by Lemma 5.3 and Corollary 5.8 any $\left(\varepsilon_{j}\right)_{j \in \mathbb{N}} \subset(0,1)$ contains a subsequence along which $c_{\varepsilon} \rightarrow c, v_{\varepsilon} \rightarrow v$ and $\kappa_{\varepsilon} \rightarrow \kappa$ a.e. in $\Omega \times(0, T)$, a similar argument as that leading to (5.21) applies to yield (5.22).

\subsection{Proof of Theorem 1.1}

As a final preparation, let us state the following elementary observation.

Lemma 5.10 Let $N \geq 1, G \subset \mathbb{R}^{N}$ be measurable, and $\left(u_{j}\right)_{j \in \mathbb{N}} \subset L^{2}(G)$ and $\left(w_{j}\right)_{j \in \mathbb{N}} \subset L^{\infty}(G)$ be such that as $j \rightarrow \infty$ we have $u_{j} \rightarrow u$ in $L^{2}(G)$ and $w_{j} \rightarrow w$ a.e. in $G$ with some $u \in L^{2}(G)$ and $w \in L^{\infty}(G)$, and such that $\sup _{j \in \mathbb{N}}\left\|w_{j}\right\|_{L^{\infty}(G)}<\infty$. Then $u_{j} w_{j} \rightarrow u w$ in $L^{2}(G)$ as $j \rightarrow \infty$. 
Proof. We may take $C_{1}>0$ such that $\left|w_{j}\right| \leq C_{1}$ in $G$ for all $j \in \mathbb{N}$, and then estimate, using the elementary inequality $(A+B)^{2} \leq 2\left(A^{2}+B^{2}\right)$ for $A \geq 0$ and $B \geq 0$,

$$
\begin{aligned}
\int_{G}\left(u_{j} w_{j}-u w\right)^{2} & \leq 2 \int_{G}\left(u_{j}-u\right)^{2} w_{j}^{2}+2 \int_{G} u^{2}\left(w_{j}-w\right)^{2} \\
& \leq 2 C_{1}^{2} \int_{G}\left(u_{j}-u\right)^{2}+2 \int_{G} u^{2}\left(w_{j}-w\right)^{2} \quad \text { for all } j \in \mathbb{N} .
\end{aligned}
$$

Here the first integral vanishes in the limit $j \rightarrow \infty$ by assumption, whereas in the second we apply the dominated convergence theorem along with the uniform majorization $u^{2}\left(w_{j}-w\right)^{2} \leq 4 C_{1}^{2} u^{2} \in L^{1}(G)$ to infer that also $\int_{G} u^{2}\left(w_{j}-w\right)^{2} \rightarrow 0$ as $j \rightarrow \infty$.

On the basis of the above estimates, we can now construct a global weak solution of (1.1) as a limit of an appropriate sequence of solutions to (2.1).

Proof of Theorem 1.1. First, Lemma 5.9 and Lemma 5.1 along with a standard extraction argument involving suitable diagonal sequences allows us to find $\left(\varepsilon_{j}\right)_{j \in \mathbb{N}} \subset(0,1)$ such that $\varepsilon_{j} \searrow 0$ as $j \rightarrow \infty$ and

$$
c_{\varepsilon} \rightarrow c \quad \text { in } L_{l o c}^{2}(\bar{\Omega} \times[0, \infty)) \quad \text { and a.e. in } \Omega \times(0, \infty)
$$

as well as

$$
\nabla \sqrt{1+c_{\varepsilon}} \rightarrow \nabla \sqrt{1+c} \quad \text { in } L_{l o c}^{\frac{4}{3}}(\bar{\Omega} \times[0, \infty))
$$

as $\varepsilon=\varepsilon_{j} \searrow 0$ with some nonnegative $c \in L_{l o c}^{2}(\bar{\Omega} \times[0, \infty))$ fulfilling $\nabla \sqrt{1+c} \in L_{l o c}^{\frac{4}{3}}(\bar{\Omega} \times[0, \infty))$. In view of Corollary 5.8, Lemma 5.4, Lemma 3.3, Lemma 3.5, Lemma 3.6, and Lemma 3.7 we may next pass to a subsequence to obtain nonnegative functions

$$
\left\{\begin{array}{l}
v \in L^{\infty}(\Omega \times(0, \infty)) \cap L_{l o c}^{2}\left([0, \infty) ; W^{1,2}(\Omega)\right), \\
l \in L^{\infty}\left((0, \infty) ; W^{1,2}(\Omega)\right), \\
y_{1} \in L^{\infty}(\Omega \times(0, \infty), \\
y_{2} \in L^{\infty}(\Omega \times(0, \infty) \quad \text { and } \\
\kappa \in L^{\infty}(\Omega \times(0, \infty)
\end{array}\right.
$$

such that

$$
\begin{aligned}
& v_{\varepsilon} \rightarrow v \quad \text { in } L_{l o c}^{2}(\bar{\Omega} \times[0, \infty)) \text { and a.e. in } \Omega \times(0, \infty), \\
& \nabla v_{\varepsilon} \rightarrow \nabla v \quad \text { in } L_{l o c}^{2}(\bar{\Omega} \times[0, \infty)), \\
& l_{\varepsilon} \rightarrow l \quad \text { in } L_{l o c}^{2}(\bar{\Omega} \times[0, \infty)) \text { and a.e. in } \Omega \times(0, \infty), \\
& \nabla l_{\varepsilon} \rightarrow \nabla l \quad \text { in } L_{l o c}^{2}(\bar{\Omega} \times[0, \infty)), \\
& y_{1 \varepsilon} \rightarrow y_{1} \quad \text { in } L_{l o c}^{2}(\bar{\Omega} \times[0, \infty)) \text { and a.e. in } \Omega \times(0, \infty) \text {, } \\
& y_{2 \varepsilon} \rightarrow y_{2} \quad \text { in } L_{l o c}^{2}(\bar{\Omega} \times[0, \infty)) \text { and a.e. in } \Omega \times(0, \infty) \quad \text { and } \\
& \kappa_{\varepsilon} \rightarrow \kappa \quad \text { in } L_{l o c}^{2}(\bar{\Omega} \times[0, \infty)) \quad \text { and a.e. in } \Omega \times(0, \infty)
\end{aligned}
$$


as $\varepsilon=\varepsilon_{j} \searrow 0$. A final extraction on the basis of Lemma 5.9 ensures that we may moreover assume that

$$
\frac{\kappa_{\varepsilon} \sqrt{1+c_{\varepsilon}}}{1+c_{\varepsilon} v_{\varepsilon}} \rightarrow \frac{\kappa \sqrt{1+c}}{1+c v} \quad \text { in } L_{l o c}^{4}(\bar{\Omega} \times[0, \infty))
$$

as $\varepsilon=\varepsilon_{j} \searrow 0$. In view of Lemma 5.10, the convergence properties (5.24), (5.27), and (5.33) in conjunction with the uniform bound $\frac{\kappa_{\varepsilon} v_{\varepsilon}}{1+v_{\varepsilon}} \leq K_{\kappa}$, as asserted by Lemma 3.7, imply that also

$$
\frac{\kappa_{\varepsilon} v_{\varepsilon}}{1+v_{\varepsilon}} c_{\varepsilon} \rightarrow \frac{\kappa v}{1+v} c \quad \text { in } L_{l o c}^{2}(\bar{\Omega} \times[0, \infty)),
$$

and likewise (5.24) and (5.29) guarantee that

$$
\frac{1}{1+c_{\varepsilon} l_{\varepsilon}} c_{\varepsilon} \rightarrow \frac{1}{1+c l} c \quad \text { in } L_{l o c}^{2}(\bar{\Omega} \times[0, \infty))
$$

as $\varepsilon=\varepsilon_{j} \searrow 0$.

Now given $T>0$ and $\varphi \in C_{0}^{\infty}(\bar{\Omega} \times[0, T))$, testing the first equation in (2.1) against $\varphi$ we obtain

$$
\begin{aligned}
-\int_{0}^{T} \int_{\Omega} c_{\varepsilon} \partial_{t} \varphi-\int_{\Omega} c_{0 \varepsilon} \varphi(\cdot, 0)= & -\varepsilon \int_{0}^{T} \int_{\Omega} \nabla c_{\varepsilon} \cdot \nabla \varphi-2 \int_{0}^{T} \int_{\Omega} \frac{\kappa_{\varepsilon} \sqrt{1+c_{\varepsilon}}}{1+c_{\varepsilon} v_{\varepsilon}} \nabla \sqrt{1+c_{\varepsilon}} \cdot \nabla \varphi \\
& +\int_{0}^{T} \int_{\Omega} \frac{\kappa_{\varepsilon} v_{\varepsilon}}{1+v_{\varepsilon}} c_{\varepsilon} \nabla v_{\varepsilon} \cdot \nabla \varphi+\int_{0}^{T} \int_{\Omega} \frac{1}{1+c_{\varepsilon} l_{\varepsilon}} c_{\varepsilon} \nabla l_{\varepsilon} \cdot \nabla \varphi \\
& +\mu_{c} \int_{0}^{T} \int_{\Omega} c_{\varepsilon} \varphi-\mu_{c} \int_{0}^{T} \int_{\Omega} c_{\varepsilon}^{2} \varphi-\mu_{c} \eta_{1} \int_{0}^{T} \int_{\Omega} c_{\varepsilon} v_{\varepsilon} \varphi \\
& -\varepsilon \int_{0}^{T} \int_{\Omega} c_{\varepsilon}^{\theta} \varphi
\end{aligned}
$$

for all $\varepsilon \in(0,1)$. Here by $(5.24),(2.4)$, and $(5.27)$,

$$
-\int_{0}^{T} \int_{\Omega} c_{\varepsilon} \partial_{t} \varphi-\int_{\Omega} c_{0 \varepsilon} \varphi(\cdot, 0) \rightarrow-\int_{0}^{T} \int_{\Omega} c \partial_{t} \varphi-\int_{\Omega} c_{0} \varphi(\cdot, 0)
$$

and

$$
\mu_{c} \int_{0}^{T} \int_{\Omega} c_{\varepsilon} \varphi-\mu_{c} \int_{0}^{T} \int_{\Omega} c_{\varepsilon}^{2} \varphi-\mu_{c} \eta_{1} \int_{0}^{T} \int_{\Omega} c_{\varepsilon} v_{\varepsilon} \varphi \rightarrow \mu_{c} \int_{0}^{T} \int_{\Omega} c \varphi-\mu_{c} \int_{0}^{T} \int_{\Omega} c^{2} \varphi-\mu_{c} \eta_{1} \int_{0}^{T} \int_{\Omega} c v \varphi
$$

as $\varepsilon=\varepsilon_{j} \searrow 0$, whereas combining (5.34) with (5.25), (5.35) with (5.28) and (5.36) with (5.30), respectively, shows that

$$
-2 \int_{0}^{T} \int_{\Omega} \frac{\kappa_{\varepsilon} \sqrt{1+c_{\varepsilon}}}{1+c_{\varepsilon} v_{\varepsilon}} \nabla \sqrt{1+c_{\varepsilon}} \cdot \nabla \varphi \rightarrow-2 \int_{0}^{T} \int_{\Omega} \frac{\kappa \sqrt{1+c}}{1+c v} \nabla \sqrt{1+c} \cdot \nabla \varphi
$$

and

$$
\int_{0}^{T} \int_{\Omega} \frac{\kappa_{\varepsilon} v_{\varepsilon}}{1+v_{\varepsilon}} c_{\varepsilon} \nabla v_{\varepsilon} \cdot \nabla \varphi \rightarrow \int_{0}^{T} \int_{\Omega} \frac{\kappa v}{1+v} c \nabla v \cdot \nabla \varphi
$$


as well as

$$
\int_{0}^{T} \int_{\Omega} \frac{1}{1+c_{\varepsilon} l_{\varepsilon}} c_{\varepsilon} \nabla l_{\varepsilon} \cdot \nabla \varphi \rightarrow \int_{0}^{T} \int_{\Omega} \frac{1}{1+c l} c \nabla l \cdot \nabla \varphi
$$

as $\varepsilon=\varepsilon_{j} \searrow 0$. Finally, in order to estimate the artificial terms in (5.37) we invoke once again Lemma 4.1 to find $C_{1}(T)>0$ and $C_{2}(T)>0$ such that

$$
\varepsilon \int_{0}^{T} \int_{\Omega} \frac{\left|\nabla c_{\varepsilon}\right|^{2}}{c_{\varepsilon}} \leq C_{1}(T)
$$

and

$$
\varepsilon \int_{0}^{T} \int_{\Omega} c_{\varepsilon}^{\theta} \ln \left(2+c_{\varepsilon}\right) \leq C_{2}(T)
$$

for all $\varepsilon \in(0,1)$. Therefore, by the Cauchy-Schwarz inequality and Lemma 3.2 we see that

$$
\begin{aligned}
\left|-\varepsilon \int_{0}^{T} \int_{\Omega} \nabla c_{\varepsilon} \cdot \nabla \varphi\right| & \leq \varepsilon\left(\int_{0}^{T} \int_{\Omega} \frac{\left|\nabla c_{\varepsilon}\right|^{2}}{c_{\varepsilon}}\right)^{\frac{1}{2}} \cdot\left(\int_{0}^{T} \int_{\Omega} c_{\varepsilon}\right)^{\frac{1}{2}} \cdot\|\nabla \varphi\|_{L^{\infty}(\Omega \times(0, T))} \\
& \leq\left(C_{1}(T) m T \varepsilon\right)^{\frac{1}{2}} \cdot\|\nabla \varphi\|_{L^{\infty}(\Omega \times(0, T))} \\
& \rightarrow 0 \quad \text { as } \varepsilon \searrow 0 .
\end{aligned}
$$

In order to derive a similar conclusion concerning the last term in (5.37), given $\delta>0$ we fix $K>0$ large such that $\frac{C_{2}(T)}{\ln (2+K)}<\frac{\delta}{2}$ and then choose $\varepsilon_{0} \in(0,1)$ small enough satisfying $K^{\theta}|\Omega| T \varepsilon_{0}<\frac{\delta}{2}$. Then for each $\varepsilon \in\left(0, \varepsilon_{0}\right)$ we have

$$
\begin{aligned}
\varepsilon \int_{0}^{T} \int_{\Omega} c_{\varepsilon}^{\theta} & =\varepsilon \int_{0}^{T} \int_{\Omega} \chi_{\left\{c_{\varepsilon} \leq K\right\}} c_{\varepsilon}^{\theta}+\varepsilon \int_{0}^{T} \int_{\Omega} \chi_{\left\{c_{\varepsilon}>K\right\}} c_{\varepsilon}^{\theta} \\
& \leq K^{\theta}|\Omega| T \varepsilon+\frac{\varepsilon}{\ln (2+K)} \int_{0}^{T} \int_{\Omega} c_{\varepsilon}^{\theta} \ln \left(2+c_{\varepsilon}\right) \\
& <\delta
\end{aligned}
$$

by (5.44). This clearly implies that also

$$
-\varepsilon \int_{0}^{T} \int_{\Omega} c_{\varepsilon}^{\theta} \varphi \rightarrow 0 \quad \text { as } \varepsilon \searrow 0,
$$

whence collecting (5.37)-(5.42) and (5.45) we infer that indeed (5.1) holds.

The verification of (5.2)-(5.6) can be achieved in a straighforward manner by repeatedly making use of (5.24), (5.27)-(5.33) and (2.4) as well as Lemma 5.4.

Finally, the boundedness properties of $c, v, y_{1}, y_{2}$ and $\kappa$ listed in (1.8) are immediate consequences of Fatou's lemma in conjunction with (5.24), Lemma 3.2 and (5.26). The claimed estimates for $l$ easily result from (5.26) and Lemma 3.5, because by applying the latter along a further subsequence we clearly also have $l_{\varepsilon} \rightarrow l$ in $L_{l o c}^{2}\left([0, \infty) ; W^{2,2}(\Omega)\right)$. 


\section{Discussion}

We proved the global existence of a weak solution to the multiscale model set up in [22]. The uniqueness in the function spaces considered here is still to be addressed.

The numerical simulations of our model, performed with the parameter values and data in [22] and with the same numerical scheme (implicit-explicit finite differences) show that the cell density in the tumor can regrow after the decay due to dispersal and even exceed its initial value in that region. More precisely, we fix $f(c, l):=\frac{D_{K}}{1+c l}$ and consider the model

$$
\left\{\begin{array}{l}
\partial_{t} c=\nabla \cdot\left(D_{C} \frac{\kappa}{1+c v} \nabla c\right)-\nabla \cdot\left(\frac{D_{H} \kappa v}{1+v} c \nabla v\right)-\nabla \cdot(f(c, l) c \nabla l)+\mu_{c} c\left(1-c-\eta_{1} v\right) \\
\partial_{t} v=-\delta_{v} c v+\mu_{v} v\left(1-\eta_{2} c-v\right) \\
\partial_{t} l=\alpha \Delta l+\delta_{l} c v-\beta l \\
\partial_{\theta} y_{1}=k_{1}\left(1-y_{1}-y_{2}\right) v-k_{-1} y_{1} \\
\partial_{\theta} y_{2}=k_{2}\left(1-y_{1}-y_{2}\right) l-k_{-2} y_{2} \\
\partial_{\theta} \kappa=-q \kappa+\frac{M y_{1}(\theta-\tau)}{1+y_{2}(\theta-\tau)}
\end{array}\right.
$$

where the dimensionless time variable $t$ is used on the macroscale, while $\theta=\frac{t}{0.01}$ represents time on the microscale (recall that the microscopic subcellular processes are much faster than those on the macrolevel). Using $\Omega=(0,1) \subset \mathbb{R}, \tau=4$ and

$$
\begin{aligned}
& D_{C}=10^{-3}, \quad D_{H}=1, \quad D_{K}=0.5, \quad \mu_{c}=1, \quad \eta_{1}=0.05, \quad \delta_{v}=10, \quad \mu_{v}=0.3, \quad \eta_{2}=0.9 \text {, } \\
& \delta_{l}=0.05, \quad \beta=0.15, \quad k_{1}=2, \quad k_{-1}=0.06, \quad k_{2}=0.31, \quad k_{-2}=0.048, \quad q=3, \quad M=1 \text {, }
\end{aligned}
$$

we compare the cases where the diffusive constant $\alpha$ of the proteolytic residuals is either $\alpha=0.1$ or $\alpha=1$. All other data and parameters are the same as in [22, Section 5], except for $c_{0}$, which is taken here smaller than 1. The numerical simulations of (6.1) are presented in Figure 1. Notice that a slower diffusion of the proteolytic residuals causes a density increase in the original tumor, which reaches clearly above the initial value $c_{0}$ and also the (rescaled) carrying capacity, as the cancer cells tend to agglomerate in the region with the higher value of the chemoattractant. However, when using the considerably larger diffusion coefficient $\alpha$ this effect disappears or is minimal and the invasion is enhanced.

The present analytical result also holds if the contractivity function is adequately involved in the chemotaxis term as well. This accounts for changes in the chemotactic behavior due to modifications of the intracellular state. The latter are seen as a consequence of enhanced (or reduced) integrin binding to proteolytic residuals, according to their availability in the peritumoral environment. In this case, we compare the previous model (6.1) with the same model, but with the choice $f(c, l, \kappa):=\frac{D_{K} \kappa}{1+c l}$. Also, we choose $l_{0}$ and $y_{20}$ in a different way than it has been done in [22, Section 5], to which we also refer for details about the numerical scheme and the parameter ranges. This new choice of data allows for a more realistic dynamics of $l$ : there are no proteolytic rests left inside the original tumor and their concentration increases as the tumor outmost boundary (with the most aggressive cells) is approached, to slowly decrease again towards the region where no cancer cells arrived yet and hence were not able to 


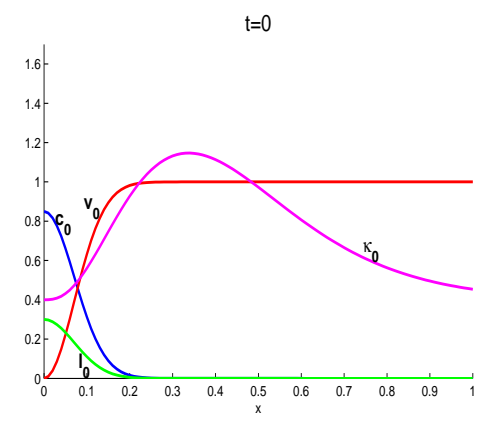

(a)

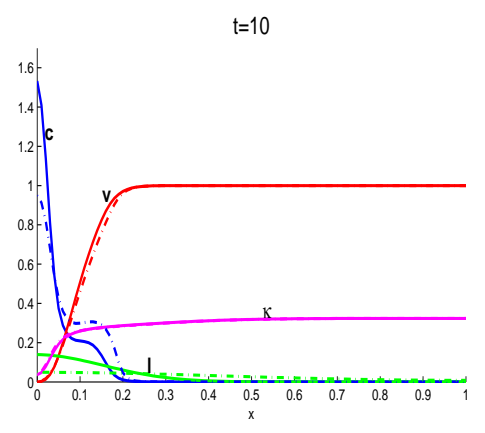

(b)

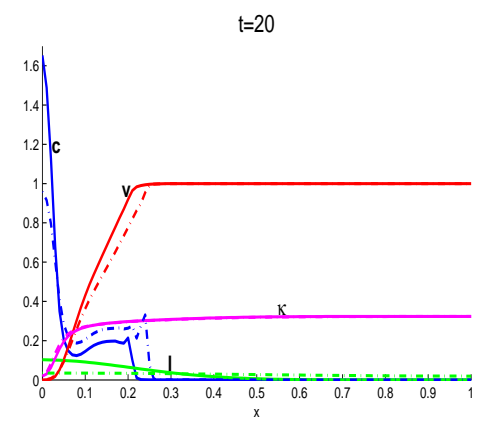

(c)

Figure 1: Evolution of tumor cell density (blue), ECM fiber density (red), concentration of proteolytic rests (green), and contractivity function (purple) for (6.1) with $f(c, l)=\frac{D_{K}}{1+c l}$ in the cases $\alpha=0.1$ (solid line) and $\alpha=1$ (dash-dot line).

dissolve the ECM fibers. Figure 2 illustrates some time snapshots for this comparison. The situation without contractivity in the chemotaxis term seems to predict a more aggressive invasion, along with a decay in the original tumor, indicating that many of the cells therein have become highly motile. As expected, the presence of the contractivity function is observed to slow down the overall invasion.

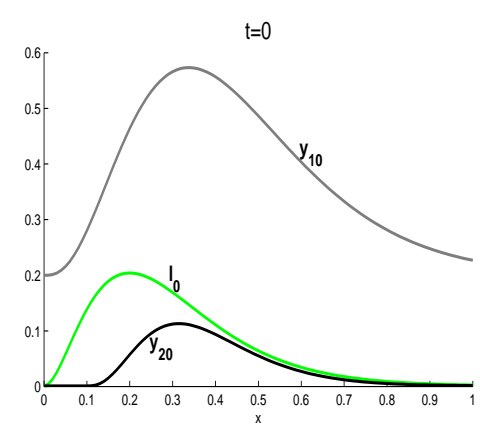

(a)

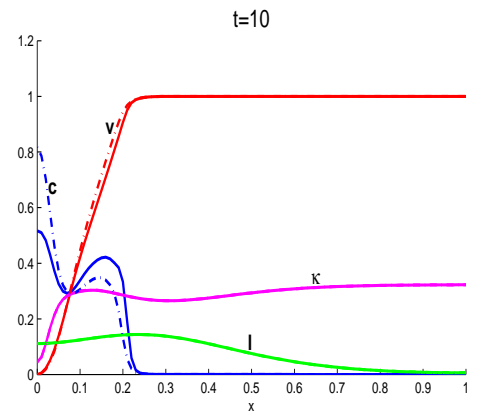

(b)

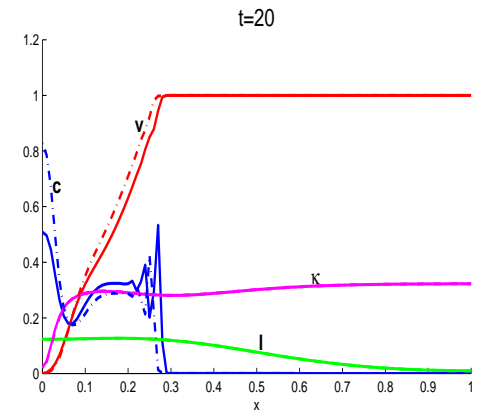

(c)

Figure 2: Evolution of tumor cell density (blue), ECM fiber density (red), concentration of proteolytic rests (green), and contractivity function (purple) for (6.1) with $\alpha=0.1$ in the cases $f(c, l)=\frac{D_{K}}{1+c l}$ (solid line) and $f(c, l, \kappa)=\frac{D_{K} \kappa}{1+c l}$ (dash-dot line). Initial conditions are shown at $t=0$, where $c_{0}, v_{0}$ and $\kappa_{0}$ are the same as in Figure 1(a).

Furthermore, as in [22], the model analyzed in this paper accounts for multiscality by incorporating the integrin binding dynamics on the microscale and coupling it with the macroscale by way of the contractivity function $\kappa$. The subcellular dynamics are thus included in a very simplified manner, in order to preserve the model parsimony and maintain a low computational effort. However, if a 
higher level of detail is wanted for the subcellular dynamics (for instance accounting for one or more intracellular signaling pathways relevant for proliferation, polarization, MDE production etc., which are known to be initiated by simple integrin binding on the cell surface, see e.g., [13, 14]), then the ODE system in (1.1) can be easily supplemented with the ODEs for the corresponding mass action kinetics and the well posedness proof is expected to apply to the new (much larger) system as well.

\section{References}

[1] Alt., H.W.: Lineare Funktionalanalysis (5th ed.). Springer-Verlag, Berlin/Heidelberg, 2006

[2] Anderson, A.R.A., Chaplain, M.A.J., Newman, E.L., Steele, R.J.C., And Thompson, A.M.: Mathematical modeling of tumor invasion and metastasis. J. of Theoretical Medicine 2, 129-154 (2000)

[3] Blanchet, A., Dolbeault, J., And Perthame, B.: Two-dimensional Keller-Segel model: optimal critical mass and qualitative properties of the solutions. Electron. J. Differential Equations 2006, No. 44, 33pp. (2006)

[4] CieŚlak, T., And Stinner, C.: Finite-time blowup and global-in-time unbounded solutions to a parabolic-parabolic quasilinear Keller-Segel system in higher dimensions. J. Differential Equations 252 (10), 5832-5851 (2012)

[5] Cieślak, T., AND Winkler, M.: Finite-time blow-up in a quasilinear system of chemotaxis. Nonlinearity 21, 1057-1076 (2008)

[6] Corrias, L., Perthame, B., And ZaAg, H.: Global Solutions of Some Chemotaxis and Angiogenesis Systems in High Space Dimensions. Milan J. Math. 72, 1-28 (2004)

[7] van Der Flier, A., And Sonnenberg, A.: Function and interactions of integrins. Cell Tissue Res. 305, 285-298 (2001)

[8] Hanahan, D., and Weinberg, R.A.: Hallmarks of cancer: the next generation. Cell 144 (5), 646-674 (2011)

[9] Hatzikirou, H., Basanta, D., Simon, M., Schaller, K., And Deutsch, A.: 'Go or Grow': the key to the emergence of invasion in tumour progression? Math. Med. Biol. 29, 49-65 (2012)

[10] Herrero, M.A., And Velázquez, J.J.L.: A blow-up mechanism for a chemotaxis model. Ann. Scuola Norm. Sup. Pisa Cl. Sci. 24, 633-683 (1997)

[11] Hieber, M., AND Prüss, J.: Heat kernels and maximal $L^{p}-L^{q}$ estimates for parabolic evolution equations. Comm. Partial Differential Equations 22 (9-10), 1647-1669 (1997)

[12] Hillen, T., Painter, K.J., And Winkler, M.: Convergence of a cancer invasion model to a logistic chemotaxis model. Math. Models Methods Appl. Sci. 23, 165-198 (2013)

[13] Hood, J.D., And Cheresh, D.A.: Role of integrins in cell invasion and migration. Nature Rev. Cancer 2, 91-100 (2002) 
[14] Huttenlocher, A., And Horwitz A.R.: Integrins in cell migration. Cold Spring Harb. Perspect. Biol. 3, a005074 (2011)

[15] Kelkel, J., And Surulescu, C.: A multiscale approach to cell migration in tissue networks. Math. Models Methods Appl. Sci. 22, 1150017 (2012)

[16] Ladyženskaja, O. A., Solonnikov, V. A., And Ural'Ceva, N. N.: Linear and Quasi-Linear Equations of Parabolic Type. Amer. Math. Soc. Transl., Vol. 23, Providence, RI, 1968

[17] Liţcanu, G., And Morales-Rodrigo, C.: Asymptotic behavior of global solutions to a model of cell invasion. Math. Models Methods Appl. Sci. 20, 1721-1758 (2010)

[18] Lorenz, T., And Surulescu, C., On a class of multiscale cancer cell migration models: wellposedness in less regular function spaces. preprint TU Kaiserslautern (2013), submitted

[19] Macklin, P., McDougall, S., Anderson, A.R.A., Chaplain, M.A.J., Cristini, V., And LOWEngRub, J.: Multiscale modelling and nonlinear simulation of vascular tumour growth. J. Math. Biol. 58, 765-798 (2009)

[20] Mallet, D.G., And Pettet, G.J.: A Mathematical Model of Integrin-Mediated Haptotactic Cell Migration. Bull. Math. Biol. 68, 231-253 (2006)

[21] Marciniak-Czochra, A., And Ptashnyk, M.: Boundedness of solutions of a haptotaxis model. Math. Models Methods Appl. Sci. 20, 449-476 (2010)

[22] Meral, G., Stinner, C., And Surulescu, C.: On a multiscale model involving cell contractivity and its effects on tumor invasion. preprint TU Kaiserslautern (2013), submitted

[23] Meral, G., And Surulescu, C.: Mathematical modelling, analysis and numerical simulations for the influence of heat shock proteins on tumour invasion. J. Math. Anal. Appl. (2013), in print

[24] Mizoguchi, N., AND Winkler, M.: Is aggregation a generic phenomenon in the twodimensional Keller-Segel system? Preprint

[25] Osaki, K., Tsujikawa, T., Yagi, A., And Mimura, M.: Exponential attractor for a chemotaxis-growth system of equations. Nonlinear Anal. TMA 51, 119-144 (2002)

[26] Porzio, M.M., And Vespri, V.: Holder Estimates for Local Solutions of Some Doubly Nonlinear Degenerate Parabolic Equations. J. Differential Equations 103, 146-178 (1993)

[27] Ramis-Conde, I., Drasdo, D., Anderson, A.R.A., and Chaplain, M.A.J.: Modeling the Influence of the E-Cadherin- $\beta$ Catenin Pathway in Cancer Cell Invasion: A Multiscale Approach. Biophys. J. 95, 155-165 (2008)

[28] Ramis-Conde, I., Chaplain, M.A.J., Anderson, A.R.A., And Drasdo, D.: Multi-scale modelling of cancer cell intravasation: the role of cadherins in metastasis. Phys. Biol. 6016008 (2009)

[29] TAO, Y.: Global existence of classical solutions to a combined chemotaxis-haptotaxis model with logistic source. J. Math. Anal. Appl. 354, 60-69 (2009) 
[30] TAO, Y.: Global existence for a haptotaxis model of cancer invasion with tissue remodeling. Nonlinear Anal. Real World Appl. 12, 418-435 (2011)

[31] TaO, Y., And CuI, C.: A density-dependent chemotaxis-haptotaxis system modeling cancer invasion. J. Math. Anal. Appl. 367, 612-624 (2010)

[32] TAO, Y., AND WANG, M.: Global solution for a chemotactic-haptotactic model of cancer invasion. Nonlinearity 21, 2221-2238 (2008)

[33] TaO, Y., And Wang, M.: A combined chemotaxis-haptotaxis system: the role of logistic source. SIAM J. Math. Anal. 41, 1533-1558 (2009)

[34] TaO, Y., And WinkleR, M.: A haptotaxis-chemotaxis model: the role of nonlinear diffusion and logistic source. SIAM J. Math. Anal. 43, 685-704 (2011)

[35] TaO, Y., AND Winkler, M.: Boundedness and stabilization in a multi-dimensional chemotaxishaptotaxis model. Proc. Roy. Soc. Edinburgh Sect. A, to appear

[36] TAO, Y., AND Winkler, M.: Energy-type estimates and global solvability in a two-dimensional chemotaxis-haptotaxis model with remodeling of non-diffusible attractant. Preprint

[37] Temam, R.: Navier-Stokes equations. Theory and numerical analysis. Studies in Mathematics and its Applications. Vol. 2. North-Holland, Amsterdam, 1977

[38] Walker, C., And WebB, G.F.: Global existence of classical solutions for a haptotaxis model. SIAM J. Math. Anal. 38, 1694-1713 (2007)

[39] Wang, Z., Bordas, V., Sagotsky, J., and Deisboeck, T.S.: Simulating Cancer Growth with Agent-Based Models, in Deisboeck, T.S. and Stamatakos, G.S. (eds.): Multiscale Cancer Modeling. Chapman \& Hall/CRC Press, Boca Raton, 2011

[40] Webb, S.D., Sherratt, J.A., And Fish, R.G.: Alterations in proteolytic activity at low $p H$ and its association with invasion: A theoretical model. Clinical Exp. Metastasis 17, 397-407 (1999)

[41] Winkler, M.: Boundedness in the higher-dimensional parabolic-parabolic chemotaxis system with logistic source. Comm. Partial Differential Equations 35, 1516-1537 (2010)

[42] Winkler, M.: Global large-data solutions in a chemotaxis-(Navier-)Stokes system modeling cellular swimming in fluid drops. Comm. Partial Differential Equations 37 (2), 319-351 (2012)

[43] Winkler, M: Finite-time blow-up in the higher-dimensional parabolic-parabolic Keller-Segel system. J. Math. Pures Appl., to appear, arXiv:1112.4156v1

[44] Zhang, L., Wang, Z., Sagotsky, J.A., And Deisboeck, T.S.: Multiscale agent-based cancer modeling. J. Math. Biol. 58, 545-559 (2009) 